\title{
DIRICHLET SERIES AND AUTOMORPHIC FUNCTIONS ASSOCIATED TO A QUADRATIC FORM
}

\author{
MANFRED PETER
}

\begin{abstract}
Starting from the reciprocity law for Gaussian sums attached to an integral quadratic form we prove functional equations for a new kind of Dirichlet series in two variables. For special values of one variable they are of Hecke type with respect to the other variable. With Weil's converse theorem we derive automorphic functions which generalize Siegel's genus invariant and the automorphic functions of Cohen and Zagier.
\end{abstract}

\section{$\S 1$. Introduction}

Several methods are known how to associate a Dirichlet series or a modular form to a quadratic form $Q$. If $Q$ is positive definite we have the Epstein zeta function and Jacobi's theta series. For $Q$ not positive definite there are also theta series but they require a more elaborate construction. If $Q$ is integral these functions contain "global" information on $Q$ since the coefficients of the Epstein zeta function (seen as an ordinary Dirichlet series) and the Fourier coefficients of the theta series are representation numbers of natural numbers by $Q$. On the other hand, a suitable linear combination of the theta series associated to representatives of the classes in the genus of $Q$ gives Siegel's genus invariant. Its Fourier coefficients can be expressed via the mass formula by the local representation densities of $Q$.

In this paper Dirichlet series and automorphic functions are constructed which contain "local" information on $Q$. Among them is Siegel's genus invariant. It turns out - maybe not surprisingly - that the reciprocity law for quadratic Gaussian sums lies at the heart of both the functional equation and the automorphic transformation law.

We continue the investigation in [5], where a pair of Dirichlet series in two variables was attached to every positive definite integral quadratic form $Q$ and their meromorphic continuation and functional equation were proved. Then two particular cases were considered:

Received April 23, 2001.

2000 Mathematics Subject Classification: 11F37 (11F66, 11E45). 
(1) The first variable was specialized to certain values so that the resulting Dirichlet series in the second variable has a functional equation of Hecke type. For the corresponding holomorphic Fourier series on the upper half plane a transformation law under $z \mapsto-1 / z$ was derived by Mellin inversion. Since only one functional equation for every Dirichlet series was available a transformation law for a congruence subgroup could not be proved. Among the Fourier series which we got in this way is Siegel's genus invariant which indeed is a modular form. Thus an immediate conjecture is that all the Fourier series are modular forms.

(2) For the quadratic form $Q(x)=x^{2}$, the corresponding Dirichlet series are - up to easily computed factors - Shintani's zeta functions for the prehomogeneous vector space of binary quadratic forms (see [7]).

The present paper generalizes [5] in two aspects: Arbitrary non-degenerate integral quadratic forms $Q$ are allowed, and the associated Dirichlet series in two variables are twisted by Dirichlet characters with prime modulus. Thus the two applications above can be developed further:

(1) With Weil's converse theorem it turns out that the holomorphic Fourier series attached to $Q$ are indeed modular forms of half-integral or integral weight (Theorem 1.1). For the special case $Q(x)=x^{2}$ they are — up to a constant factor — Cohen's modular forms [2] which have "generalized Hurwitz class numbers" as Fourier coefficients.

(2) For another special choice of the first variable the resulting Dirichlet series in the second variable has a functional equation which is not of Hecke type but with some care the proof of Weil's converse theorem still works. Thus a non-analytic $C^{\infty}$ function on the upper half plane is constructed which transforms like a modular form (Theorem 1.2). In the special case $Q(x)=x^{2}$ this function coincides with Zagier's automorphic function [13] whose holomorphic part of the Fourier expansion has Hurwitz class numbers as coefficients. This connection between Zagier's automorphic function and Shintani's zeta function gives a possible answer to a question of Datskovsky [3].

There is another connection between the Dirichlet series in two variables considered here and modular forms. In [8] Siegel constructs a vector valued function of two arguments $\tau$ and $s$ where $\tau$ lies in the upper half plane 
and, after meromorphic continuation, $s$ can take arbitrary complex values outside a finite set of poles. This function has a functional equation in $s$ and shows automorphic behaviour in $\tau$. Its Mellin transform with respect to $\tau$ is a Dirichlet series in two variables with two functional equations. In the present paper this approach is reversed. First Dirichlet series are constructed and then automorphic functions are derived from them. Nevertheless the function $\phi$ defined in Section 3 shows great similarity with Siegel's — with one exception: The summation is unsymmetric in both summation parameters whereas in Siegel's case it is symmetric in one parameter. This means for example that here we can treat class numbers for positive and negative discriminants separately whereas in Siegel's case they always appear together. It also means that here we have certain convergence problems which do not appear in Siegel's case.

T. Ueno [10], [11] investigated essentially the same Dirichlet series as in the present paper but within the framework of prehomogeneous vector spaces. The main Theorem 4.5 in [11] is essentially equivalent to Theorem 1.1 below but is proved for the wider class of half integral matrices $\mathfrak{T}$. Besides this result the papers are of conceptual interest since they give a connection between such diverse fields as the theory of prehomogeneous vector spaces and modular forms.

Denote the complex upper half plane by $\mathbb{H}$. For $z \in \mathbb{C}$, set $e(z):=e^{2 \pi i z}$. For an arbitrary Dirichlet character $\chi$, let $L(s, \chi)$ be the associated Dirichlet $L$-series. For an odd prime $q$, let $(\dot{q})$ be the Legendre character modulo $q$ and $\chi_{0}$ the principal character modulo $q$. For a Dirichlet character $\chi \neq \chi_{0}$ modulo $q$, let $\tau(\chi)$ be the Gaussian sum attached to $\chi$. Set $\epsilon_{q}:=1$ if $q \equiv 1 \bmod 4$ and $\epsilon_{q}:=i$ otherwise. For a discriminant $D \neq 0$, let $\chi_{D}$ be the corresponding Jacobi character. Let $\mathbb{Z}_{*}^{m \times m}$ be the set of regular symmetric integral $m \times m$ matrices. For $\mathfrak{T} \in \mathbb{Z}_{*}^{m \times m}$, there is a regular matrix $\mathfrak{X} \in \mathbb{R}^{m \times m}$ such that ${ }^{t} \mathfrak{X} \mathfrak{X}$ is a diagonal matrix with, say, $p$ entries 1 and $q$ entries -1 . Then $\operatorname{sign}(\mathfrak{T}):=p-q$ is independent of $\mathfrak{X}$ and called the signature of $\mathfrak{T}$. For $\mathfrak{x} \in \mathbb{R}^{m}$, define $\mathfrak{T}[\mathfrak{x}]:={ }^{t} \mathfrak{x} \mathfrak{x}$. For $h \in \mathbb{Z}, k \in \mathbb{N}$, define the Gaussian sum

$$
G(h, k ; \mathfrak{T}):=\sum_{\mathfrak{x} \bmod k} e\left(\frac{h}{k} \mathfrak{T}[\mathfrak{x}]\right) .
$$

For $b \in \mathbb{N}, n \in \mathbb{Z}$, define the "Singular Series"

$$
\mathcal{S}(s, n ; b, \mathfrak{T}):=\sum_{k \geq 1} \frac{1}{k^{s}}\left(\sum_{h \bmod b k:(h, k)=1} G(h, b k ; \mathfrak{T}) e\left(\frac{-h n}{b k}\right)\right), \quad \Re s>m+2 .
$$


It can be continued to a meromorphic function on $\mathbb{C}$. For $b=1, n>0$, the value at $s=m$ is the "Singular Series" of Hardy and Littlewood and appears also in the mass formula as the product of the local representation densities over all primes. As a Dirichlet series in $s$ it obviously contains local data about $Q(\mathfrak{x}):=\mathfrak{T}[\mathfrak{x}]$ which only depends on the genus of $Q$. The following theorem constructs modular forms out of this local data.

Theorem 1.1. Let $\mathfrak{T} \in \mathbb{Z}_{*}^{m \times m}, T:=\operatorname{det} \mathfrak{T}, \alpha \in\{1,2\}$ and $l \in \mathbb{Z}$, such that

$$
s_{\alpha l}:=\frac{m}{2}+\frac{1}{2}(-1)^{\alpha-1} \operatorname{sign}(\mathfrak{T})+2 l>\sigma(m),
$$

where $\sigma(m):=(m+3) / 2$ for $m$ odd and $\sigma(m):=(m+2) / 2$ for $m$ even. Set $\kappa_{\alpha l}:=2 s_{\alpha l}-m$. Set $b:=1, \mathfrak{S}:=\mathfrak{T}$, or $b:=4|T|, \mathfrak{S}:=|T| \mathfrak{T}^{-1}$. Set $S:=\operatorname{det} \mathfrak{S}$. For $z \in \mathbb{H}$, define

$$
\begin{aligned}
\Theta_{\alpha l}(z ; b, \mathfrak{S}):= & b^{s_{\alpha l}}|S|^{1 / 2} 2^{m / 2-\kappa_{\alpha l} / 2} \pi^{-\kappa_{\alpha l} / 2}(-1)^{l} \Gamma\left(\frac{\kappa_{\alpha l}}{2}\right) \\
& +\sum_{n \geq 1} n^{\kappa_{\alpha l} / 2-1} \mathcal{S}\left(s_{\alpha l},(-1)^{\alpha-1} n ; b, \mathfrak{S}\right) e(n z) .
\end{aligned}
$$

Then $\Theta_{\alpha l}(z ; b, \mathfrak{S})$ is a modular form of weight $\kappa_{\alpha l} / 2$ with respect to $\Gamma_{0}(4|T|)$ and

a) the theta multiplier system and the character $\chi_{4|S|}$ if $m$ is odd,

b) the character $\chi_{(-1)^{m / 2} 4 T}$ if $m$ is even.

The smaller $s_{\alpha l}$ is the more interesting are the values of $\mathcal{S}$. For $m$ odd, $(m+3) / 2$ is a critical value. The following theorem states automorphic behaviour in this case.

Theorem 1.2. Let $m$ be odd, $\mathfrak{T} \in \mathbb{Z}_{*}^{m \times m}$ and $T:=\operatorname{det} \mathfrak{T}$. Set $\lambda:=$ $(1 \pm \operatorname{sign}(\mathfrak{T})) / 4$, where the sign is chosen so that $\lambda \in \mathbb{Z}$. Set $b:=1$, $\mathfrak{S}:=\mathfrak{T}$, or $b:=4|T|$, $\mathfrak{S}:=|T| \mathfrak{T}^{-1}$. Set $S:=\operatorname{det} \mathfrak{S}$. For $z=x+i y \in \mathbb{H}$, define

$$
\begin{aligned}
\mathcal{F}(z ; b, \mathfrak{S}):= & \pi^{-1} 2^{(m-5) / 2} b^{(m+3) / 2}|S|^{1 / 2}(-1)^{\lambda+1} \\
& +\sum_{n \geq 1} \mathcal{S}\left(\frac{m+3}{2},(-1)^{(m+1) / 2} \operatorname{sign}(T) n ; b, \mathfrak{S}\right) n^{1 / 2} e(n z) \\
& +\frac{3}{(2 \pi)^{2}} b^{(m+3) / 2} y^{-1 / 2}
\end{aligned}
$$




$$
\times\left[\prod_{p \mid 2 T}\left(1-p^{-2}\right)^{-1} T_{p}(1 ; b, \mathfrak{S})+\sum_{n \geq 1} a_{n}(b, \mathfrak{S}) \gamma\left(4 \pi\left|S^{*}\right| n^{2} y\right) e\left(-\left|S^{*}\right| n^{2} z\right)\right]
$$

where the polynomials $T_{p}$, numbers $a_{n}$ and the function $\gamma$ are defined in Lemma 6.2, (7.8) and (7.4) respectively. Then $\mathcal{F}(z ; b, \mathfrak{S})$ transforms like a modular form of weight $3 / 2$ with respect to $\Gamma_{0}(4|T|)$, the theta multiplier system and the character $\chi_{4|S|}$.

Here is an overview of the proof. In Section 3, Dirichlet series $\rho_{\alpha}(s, w ; b, \mathfrak{T}, \chi)$ and Eisenstein-type series $\phi_{\alpha, \mu, \nu}(\tau, s ; b, \mathfrak{T}, \chi)$ are defined. Both have Gaussian sums as coefficients. The function $\rho_{\alpha}$ is a Mellin transform of $\phi_{\alpha, \mu, \nu}$, see equation (3.1). For technical reasons, the series $\psi_{\alpha, \mu, \nu}(\tau, s ; a, b, \mathfrak{T}, \chi)$ is introduced. It is similar to $\phi_{\alpha, \mu, \nu}$ but one of the two summations is symmetric. In Lemma 3.1 it is shown that the $\phi$ s are linear combinations of the $\psi$ s. In Section 4 , the Dirichlet series $\tau_{\alpha}(s, w ; b, \mathfrak{T}, \chi)$ are introduced. Their coefficients are numbers of solutions of quadratic congruences. The functional equation of the Hurwitz zeta function shows that $\tau_{\alpha}$ is a linear combination of the $\rho$ s (see equation (4.1)). In Section 5 the Dirichlet series $D_{\alpha}(s, w ; b, \mathfrak{T}, \chi)$ are introduced whose coefficients are values of Singular Series at complex arguments. Equations (5.1) and (5.2) show that in essence, $D_{\alpha}$ is the quotient of $\tau_{\alpha}$ and the Riemann zeta function. The Mellin transform of $\Theta_{\alpha, l}$ is $D_{\alpha}$ at a special value of $s$. Thus Weil's converse theorem can be applied to prove Theorem 1.1. The proof of Theorem 1.2 is along similar lines but a component $\tau_{\alpha}^{*}$ of $\tau_{\alpha}$ must be isolated to avoid singularities of this function.

Acknowledgements. I would like to thank Prof. H. Klingen for several useful discussions on the topic of this paper, and the referee for his suggestions for improvements.

\section{$\S 2$. A reciprocity law}

For an odd prime $q$ and a Dirichlet character $\chi \bmod q$, define

$$
\lambda(h, \chi):=\sum_{a \bmod q} \bar{\chi}(a) e\left(-\frac{h a}{q}\right), \quad h \in \mathbb{Z} .
$$

A simple calculation shows that

$$
\overline{\lambda(h, \chi)}=\chi(-1) \lambda(h, \bar{\chi}), \quad \lambda(b h, \chi)=\chi(b) \lambda(h, \chi) \text { if }(b, q)=1 .
$$


The properties of Gaussian sums imply

$$
\lambda(h, \chi)= \begin{cases}\overline{\tau(\chi)} \chi(h) & , \quad \chi \neq \chi_{0} \bmod q \\ q-1 & , \quad \chi=\chi_{0} \bmod q, q \mid h \\ -1 & , \quad \chi=\chi_{0} \bmod q, q \backslash h\end{cases}
$$

The following Gaussian sums are central to this paper: For $\mathfrak{T} \in \mathbb{Z}_{*}^{m \times m}$, $T:=\operatorname{det} \mathfrak{T}, q \backslash 2 T$ a prime, $\chi$ a Dirichlet character modulo $q, \alpha \in\{1,2\}$ and $h \in \mathbb{Z}, k \in \mathbb{N}$, define

$$
G_{\alpha}(h, k ; \mathfrak{T}, \chi):=\sum_{a \bmod k, b \bmod q: k b+(-1)^{\alpha} a q \equiv h \bmod k q} G(a, k ; \mathfrak{T}) \chi(b) .
$$

An immediate consequence is

$$
G_{\alpha}(-h, k ; \mathfrak{T}, \chi)=\chi(-1) \overline{G_{\alpha}(h, k ; \mathfrak{T}, \bar{\chi})} .
$$

Lemma 2.1. For $\mathfrak{T} \in \mathbb{Z}_{*}^{m \times m}, T:=\operatorname{det} \mathfrak{T}, q \backslash 2 T$ a prime, $\chi$ a Dirichlet character modulo $q$ and $\mathfrak{y} \in \mathbb{Z}^{m}$, we have

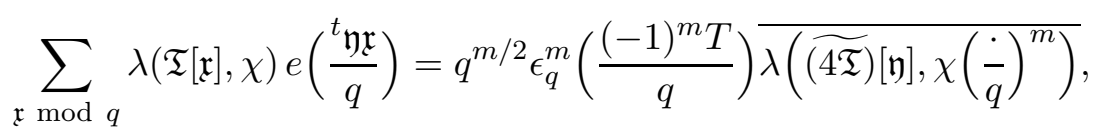

where $\widetilde{()}$ denotes inversion modulo $q$.

Proof. From [4], $\S 62$, it follows that there is some $\mathfrak{X} \in \operatorname{GL}\left(m, \mathbb{F}_{q}\right)$ with

$$
{ }^{t} \mathfrak{X T X} \equiv \operatorname{diag}\left(d_{1}, \ldots, d_{m}\right) \quad \bmod q .
$$

In particular, $T(\operatorname{det} \mathfrak{X})^{2} \equiv d_{1} \cdots d_{m} \bmod q$. Thus the left hand term $\Sigma$ in the lemma equals

$$
\Sigma=\sum_{a \bmod q} \bar{\chi}(a) \prod_{\mu=1}^{m}\left(\sum_{x_{\mu} \bmod q} e\left(-\frac{a}{q} d_{\mu} x_{\mu}^{2}+\frac{1}{q} y_{\mu}^{\prime} x_{\mu}\right)\right)
$$

where ${ }^{t} \mathfrak{X} \mathfrak{y}=: \mathfrak{y}^{\prime}={ }^{t}\left(y_{1}^{\prime}, \ldots, y_{m}^{\prime}\right)$. From the properties of the Gaussian sum associated to Legendre's character it follows that for $(a, q)=1$, the $\mu$-th factor equals

$$
\left.e\left(\frac{1}{q} \widetilde{\left(4 d_{\mu} a\right.}\right)\left(y_{\mu}^{\prime}\right)^{2}\right)\left(\frac{-a d_{\mu}}{q}\right) q^{1 / 2} \epsilon_{q}
$$


Since $\widetilde{(\mathfrak{T})} \equiv \mathfrak{X} \operatorname{diag}\left(\widetilde{\left(d_{1}\right)}, \ldots, \widetilde{\left(d_{m}\right)}\right)^{t} \mathfrak{X} \bmod q$, we have

$$
\Sigma=q^{m / 2} \epsilon_{q}^{m}\left(\frac{(-1)^{m} T}{q}\right) \sum_{a \bmod q} \bar{\chi}(a)\left(\frac{a}{q}\right)^{m} e(\widetilde{(4 a)} \widetilde{(\mathfrak{T})}[\mathfrak{y}]),
$$

which is the right hand term in the lemma.

Lemma 2.2. For $\mathfrak{T} \in \mathbb{Z}_{*}^{m \times m}, T:=\operatorname{det} \mathfrak{T}, q \backslash 2 T$ a prime, $\chi$ a Dirichlet character modulo $q$ and $h, k \in \mathbb{N}$, we have

$$
\begin{aligned}
& \sum_{\mathfrak{x} \bmod q k} \lambda(\mathfrak{T}[\mathfrak{x}], \chi) e\left(\frac{h}{k} \mathfrak{T}[\mathfrak{x}]\right) \\
& =2^{-3 m / 2} q^{-m / 2} \epsilon_{q}^{m} e^{\pi i \operatorname{sign}(\mathfrak{T}) / 4}|T|^{1 / 2-m} h^{-m / 2} k^{m / 2}\left(\frac{|T|^{m} T}{q}\right) \chi(-4|T|) \\
& \times \sum_{\mathfrak{z} \bmod 4 q^{2} h|T|} \lambda\left(|T| \mathfrak{T}^{-1}[\mathfrak{z}], \bar{\chi}\left(\frac{\cdot}{q}\right)^{m}\right) e\left(-\frac{k}{4 q^{2} h} \mathfrak{T}^{-1}[\mathfrak{z}]\right) .
\end{aligned}
$$

Proof. The lemma rests essentially on Satz 2 in [9] which is a general reciprocity law for quadratic Gaussian sums associated to quadratic forms. Let $\mathfrak{y} \in \mathbb{Z}$. Setting

$$
\mathfrak{A}:=k \mathfrak{E}, \mathfrak{B}:=2 q^{2} h \mathfrak{T}, \mathfrak{t}:=\frac{1}{q} \mathfrak{y},
$$

the theorem gives

$$
\begin{aligned}
\sum_{\mathfrak{z} \bmod k} e\left(\frac{h}{k} \mathfrak{T}[q \mathfrak{z}+\mathfrak{y}]\right) & \\
= & 2^{-3 m / 2} q^{-m} e^{\pi i \operatorname{sign}(\mathfrak{T}) / 4}|T|^{1 / 2-m} h^{-m / 2} k^{m / 2} \\
& \times \sum_{\mathfrak{z} \bmod 4 q^{2} h|T|} e\left(-\frac{k}{4 q^{2} h} \mathfrak{T}^{-1}[\mathfrak{z}]+\frac{1}{q} t_{\mathfrak{y} \mathfrak{z}}\right) .
\end{aligned}
$$

Thus the left hand term in the lemma equals

$$
\begin{aligned}
\sum_{\mathfrak{y} \bmod q} \lambda(\mathfrak{T}[\mathfrak{y}], \chi) \sum_{\mathfrak{z} \bmod k} e\left(\frac{h}{k} \mathfrak{T}[q \mathfrak{z}+\mathfrak{y}]\right) \\
=2^{-3 m / 2} q^{-m} e^{\pi i \operatorname{sign}(\mathfrak{T}) / 4}|T|^{1 / 2-m} h^{-m / 2} k^{m / 2} \\
\quad \times \sum_{\mathfrak{z} \bmod 4 q^{2} h|T|} e\left(-\frac{k}{4 q^{2} h} \mathfrak{T}^{-1}[\mathfrak{z}]\right) \sum_{\mathfrak{y} \bmod q} \lambda(\mathfrak{T}[\mathfrak{y}], \chi) e\left(\frac{1}{q} t_{\mathfrak{y} \mathfrak{z}) .}\right.
\end{aligned}
$$


Applying Lemma 2.1 to the innermost sum and using (2.1) proves the lemma.

The following reciprocity law can be regarded as the nucleus of this paper.

Proposition 2.3. For $\mathfrak{T} \in \mathbb{Z}_{*}^{m \times m}, T:=\operatorname{det} \mathfrak{T}, q \backslash 2 T$ a prime, $\chi a$ Dirichlet character modulo $q, \alpha \in\{1,2\}$ and $h, k \in \mathbb{N}$, we have

$$
\begin{aligned}
G_{\alpha}(h, k ; \mathfrak{T}, \chi)= & 2^{-3 m / 2}|T|^{1 / 2-m} e^{\pi i(-1)^{\alpha} \operatorname{sign}(\mathfrak{T}) / 4} h^{-m / 2} k^{m / 2} \\
& \times C(\chi, \alpha, \mathfrak{T}) G_{\alpha-1}\left(k, 4|T| h ;|T| \mathfrak{T}^{-1}, \bar{\chi}\left(\frac{\dot{q}}{q}\right)^{m}\right),
\end{aligned}
$$

where

$$
C(\chi, \alpha, \mathfrak{T}):=\epsilon_{q}^{m} \bar{\chi}(4|T|)\left(\frac{(-1)^{(\alpha-1) m} T|T|^{m}}{q}\right) .
$$

Proof. We distinguish three cases.

Case 1. $q \nmid h k$. A simple calculation shows that, since $q \nmid k$,

$$
G_{\alpha}(h, k ; \mathfrak{T}, \chi)=G\left((-1)^{\alpha} h q, k ; \mathfrak{T}\right) \chi(h) \bar{\chi}(k) .
$$

Satz 2 in [9] gives for $u, v \in \mathbb{N}$ the identity

$$
\begin{aligned}
& G(u, v ; \mathfrak{T})= \\
& \quad e^{\pi i \operatorname{sign}(\mathfrak{T}) / 4}|T|^{1 / 2-m} 2^{-3 m / 2} u^{-m / 2} v^{m / 2} G\left(-v, 4|T| u ;|T| \mathfrak{T}^{-1}\right) .
\end{aligned}
$$

Therefore

$$
\begin{aligned}
& G_{\alpha}(h, k ; \mathfrak{T}, \chi) \\
& =e^{\pi i(-1)^{\alpha}} \operatorname{sign}(\mathfrak{T}) / 4|T|^{1 / 2-m} 2^{-3 m / 2}(h q)^{-m / 2} k^{m / 2} \\
& \quad \times G\left((-1)^{\alpha-1} k, 4|T| h q ;|T| \mathfrak{T}^{-1}\right) \chi(h) \bar{\chi}(k) .
\end{aligned}
$$

Since $q \Varangle 4 T h$,

$$
\begin{aligned}
& G\left((-1)^{\alpha-1} k, 4|T| h q ;|T| \mathfrak{T}^{-1}\right) \\
& \quad=G\left((-1)^{\alpha-1} q k, 4|T| h ;|T| \mathfrak{T}^{-1}\right) \sum_{\mathfrak{x} \bmod q} e\left(\frac{1}{q}(-1)^{\alpha-1} 4 h k T^{2} \mathfrak{T}^{-1}[\mathfrak{x}]\right) .
\end{aligned}
$$

Using a representation of type (2.3) for $T \mathfrak{T}^{-1}$ shows that the right hand sum equals

$$
\left(\frac{(-1)^{\alpha-1} h k}{q}\right)^{m} q^{m / 2} \epsilon_{q}^{m}\left(\frac{T}{q}\right)
$$


Putting everything together gives

$$
\begin{aligned}
G_{\alpha}(h, k ; \mathfrak{T}, \chi) & \\
= & e^{\pi i(-1)^{\alpha} \operatorname{sign}(\mathfrak{T}) / 4}|T|^{1 / 2-m} 2^{-3 m / 2} h^{-m / 2} k^{m / 2} \chi(h) \bar{\chi}(k) \\
& \times\left(\frac{(-1)^{\alpha-1} h k}{q}\right)^{m} \epsilon_{q}^{m}\left(\frac{T}{q}\right) G\left((-1)^{\alpha-1} q k, 4|T| h ;|T| \mathfrak{T}^{-1}\right) .
\end{aligned}
$$

Replacing $h, k, \alpha, \mathfrak{T}, \chi$ in $(2.4)$ by $k, 4|T| h, \alpha-1,|T| \mathfrak{T}^{-1}, \bar{\chi}(\dot{q})^{m}$ gives

$$
\begin{aligned}
& G_{\alpha-1}\left(k, 4|T| h ;|T| \mathfrak{T}^{-1}, \bar{\chi}\left(\frac{\cdot}{q}\right)^{m}\right) \\
& \quad=G\left((-1)^{\alpha-1} k q, 4|T| h ;|T| \mathfrak{T}^{-1}\right) \bar{\chi}(k)\left(\frac{k}{q}\right)^{m} \chi(4|T| h)\left(\frac{4|T| h}{q}\right)^{m} .
\end{aligned}
$$

The last two equations give the statement of the proposition in Case 1.

Case 2. $q|k, q| h$. Then

$$
\begin{aligned}
G_{\alpha}(h, k ; \mathfrak{T}, \chi) & =\sum_{b \bmod q} \chi(b) G\left((-1)^{\alpha}\left(\frac{h}{q}-\frac{k}{q} b\right), k ; \mathfrak{T}\right) \\
& =q^{-m} \chi\left((-1)^{\alpha}\right) \sum_{\mathfrak{x} \bmod q k} \lambda(\mathfrak{T}[\mathfrak{x}], \bar{\chi}) e\left(\frac{(-1)^{\alpha} h}{q k} \mathfrak{T}[\mathfrak{x}]\right)
\end{aligned}
$$

Lemma 2.2 gives

$$
\begin{gathered}
G_{\alpha}(h, k ; \mathfrak{T}, \chi) \\
=q^{-m} 2^{-3 m / 2} \epsilon_{q}^{m}\left(\frac{-1}{q}\right)^{m \alpha} e^{\pi i(-1)^{\alpha} \operatorname{sign}(\mathfrak{T}) / 4}|T|^{1 / 2-m} \\
\times h^{-m / 2} k^{m / 2}\left(\frac{(-1)^{m} T}{q}\right) \chi\left((-1)^{\alpha-1}\right)\left(\frac{-1}{q}\right)^{m(\alpha-1)} \bar{\chi}(4|T|)\left(\frac{|T|}{q}\right)^{m} \\
\times \sum_{\mathfrak{x} \bmod 4 q h|T|} \lambda\left(|T| \mathfrak{T}^{-1}[\mathfrak{z}], \chi\left(\frac{\cdot}{q}\right)^{m}\right) e\left(\frac{(-1)^{\alpha-1} k}{4 q h|T|}|T| \mathfrak{T}^{-1}[\mathfrak{z}]\right) .
\end{gathered}
$$

Replacing $h, k, \alpha, \mathfrak{T}, \chi$ in $(2.6)$ by $k, 4|T| h, \alpha-1,|T| \mathfrak{T}^{-1}, \bar{\chi}(\dot{\bar{q}})^{m}$ and using (2.8) gives the statement of the proposition in Case 2.

Case 3. $q \mid h, q \backslash k$ or $q \mid k, q \backslash h$. Then $G_{\alpha}(h, k ; \mathfrak{T}, \chi)=0$. In order to see this we assume the contrary. Then there are $a$ and $b$ with $\chi(b) \neq 0$ and $k b+(-1)^{\alpha} a q \equiv h \bmod k q$. This implies $q \not\langle b$ and $k b \equiv h \bmod q$. Therefore $q|k, q| h$ or $q \nmid k, q \backslash h$ which contradicts the conditions of Case 3 . In the same way it follows that the right hand term in the proposition vanishes. 


\section{§3. Meromorphic continuation of $\rho$}

For $\mathfrak{T} \in \mathbb{Z}_{*}^{m \times m}, T:=\operatorname{det} \mathfrak{T}, b \in \mathbb{N}, q \backslash 2 T b$ a prime, $\chi$ a Dirichlet character modulo $q$ and $\alpha \in\{1,2\}$, define

$$
\rho_{\alpha}(s, w ; b, \mathfrak{T}, \chi):=\sum_{h, k \geq 1} \frac{G_{\alpha}(h, b k ; \mathfrak{T}, \chi)}{k^{s} h^{w}}, \quad \Re s>m+2, \Re w>1 .
$$

For $\mu, \nu \in\{0,1\}$, define

$$
\phi_{\alpha, \mu, \nu}(\tau, s ; b, \mathfrak{T}, \chi):=\sum_{h, k \geq 1} \frac{k^{\nu-m / 2} h^{\mu} G_{\alpha}(h, b k ; \mathfrak{T}, \chi)}{\left(h^{2}+\tau k^{2}\right)^{s}}, \quad \tau>0, \Re s>\frac{m}{4}+3 .
$$

Functions of this type were already considered in [5]. The method of that paper will be used here again. First $\rho$ is written as a Mellin transform of $\phi$. For $\Re s>m+2, \Re w>1$,

$$
\begin{aligned}
& \rho_{\alpha}(s, w ; b, \mathfrak{T}, \chi)= \\
& \quad \begin{array}{l}
\Gamma\left(\frac{s+\nu}{2}-\frac{m}{4}\right)^{-1} \Gamma\left(\frac{w+\mu}{2}\right)^{-1} \Gamma\left(\frac{s+w+\mu+\nu}{2}-\frac{m}{4}\right) \\
\quad \times \int_{0}^{\infty} \phi_{\alpha, \mu, \nu}\left(\tau, \frac{s+w+\mu+\nu}{2}-\frac{m}{4} ; b, \mathfrak{T}, \chi\right) \tau^{(s+\nu) / 2-m / 4-1} d \tau .
\end{array}
\end{aligned}
$$

This identity will be used at the end to derive the continuation of $\rho$ from that of $\phi$. In order to get the latter the following simplified version of $\phi$ is introduced. For $a \in \mathbb{N}$, define

$$
\begin{array}{r}
\psi_{\alpha, \mu, \nu}(\tau, s ; a, b, \mathfrak{T}, \chi):=\sum_{h \neq 0, k \geq 1} \frac{k^{\nu-m / 2} h^{\mu} G_{\alpha}(a h, b k ; \mathfrak{T}, \chi)}{\left(h^{2}+\tau k^{2}\right)^{s}}, \\
\tau>0, \Re s>\frac{m}{4}+3 .
\end{array}
$$

Lemma 3.1. For $\mathfrak{T} \in \mathbb{Z}_{*}^{m \times m}, T:=\operatorname{det} \mathfrak{T}, b \in \mathbb{N}, q \backslash 2 T b$ a prime, $\chi a$ Dirichlet character modulo $q, \alpha \in\{1,2\}$ and $\mu, \nu \in\{0,1\}$, we have

$$
\begin{gathered}
\left(1+(-1)^{\mu+\nu+1} e^{(-1)^{\alpha+1} \pi i \operatorname{sign}(\mathfrak{T}) / 2}\right) \phi_{\alpha, \mu, \nu}(\tau, s ; b, \mathfrak{T}, \chi) \\
=\psi_{\alpha, \mu, \nu}(\tau, s ; 1, b, \mathfrak{T}, \chi) \\
+(-1)^{\mu+\nu+1} b^{m / 2} e^{(-1)^{\alpha+1} \pi i \operatorname{sign}(\mathfrak{T}) / 4}|T|^{1 / 2-m} 2^{-3 m / 2} C(\chi, \alpha, \mathfrak{T}) \tau^{-s} \\
\times \psi_{\alpha-1, \nu, \mu}\left(\tau^{-1}, s ; b, 4|T|,|T| \mathfrak{T}^{-1}, \bar{\chi}\left(\frac{\cdot}{q}\right)^{m}\right) .
\end{gathered}
$$


Proof. From the reciprocity law in Proposition 2.3 and (2.2) it follows that the second summand on the right hand side equals

$$
\begin{aligned}
& (-1)^{\mu+\nu+1} b^{m / 2} e^{(-1)^{\alpha+1} \pi i \operatorname{sign}(\mathfrak{T}) / 4}|T|^{1 / 2-m} 2^{-3 m / 2} C(\chi, \alpha, \mathfrak{T}) \\
& \quad \times \sum_{h \neq 0, k \geq 1} \frac{k^{\mu-m / 2} h^{\nu} G_{\alpha-1}\left(b h, 4|T| k ;|T| \mathfrak{T}^{-1}, \bar{\chi}(\dot{\bar{q}})^{m}\right)}{\left(h^{2} \tau+k^{2}\right)^{s}} \\
& =(-1)^{\mu+\nu+1} e^{(-1)^{\alpha+1} \pi i \operatorname{sign}(\mathfrak{T}) / 2} \sum_{h, k \geq 1} \frac{k^{\mu} h^{\nu-m / 2} G_{\alpha}(k, b h ; \mathfrak{T}, \chi)}{\left(h^{2} \tau+k^{2}\right)^{s}} \\
& \quad+(-1)^{\mu+1} \sum_{h, k \geq 1} \frac{k^{\mu} h^{\nu-m / 2} G_{\alpha}(-k, b h ; \mathfrak{T}, \chi)}{\left(h^{2} \tau+k^{2}\right)^{s}} .
\end{aligned}
$$

Adding $\psi_{\alpha, \mu, \nu}(\tau, s ; 1, b, \mathfrak{T}, \chi)$ gives the statement of the lemma.

For the analytic continuation of $\psi$ the following facts are needed. For $\mathfrak{T} \in \mathbb{Z}_{*}^{m \times m}, T:=\operatorname{det} \mathfrak{T}, h \in \mathbb{Z}, k \in \mathbb{N}$, define

$$
A(h, k ; \mathfrak{T}):=\#\{\mathfrak{x} \bmod k \mid \mathfrak{T}[\mathfrak{x}] \equiv h \bmod k\},
$$

and for $b \in \mathbb{N}$,

$$
L(s, h ; b, \mathfrak{T}):=\sum_{k \geq 1} \frac{A(h, b k ; \mathfrak{T})}{k^{s}}, \quad \Re s>m+1 .
$$

Define

$$
\begin{aligned}
P^{*}(s ; b, \mathfrak{T}) & :=\zeta(s-m+1)^{-1} L\left(s-\frac{m}{2}+1, \chi_{(-1)^{m / 2} 4 T b^{2}}\right), \\
P(s ; b, \mathfrak{T}) & :=P^{*}(s ; b, \mathfrak{T}) \prod_{p \mid 2 T b}\left(1-p^{m-2 s}\right)(2 s-m-2),
\end{aligned}
$$

if $m$ is even and

$$
\begin{aligned}
P^{*}(s ; b, \mathfrak{T}) & :=P(s ; b, \mathfrak{T}):= \\
\zeta(s-m+1)^{-1} \zeta(2 s-m+1) & \prod_{p \mid 2 T b}\left(1-p^{m-1-2 s}\right)(2 s-m-1),
\end{aligned}
$$

if $m$ is odd.

Lemma 3.2. Let $\mathfrak{T} \in \mathbb{Z}_{*}^{m \times m}, T:=\operatorname{det} \mathfrak{T}, b \in \mathbb{N}$. 
a) For $h \in \mathbb{Z} \backslash\{0\}$, the function $L(s, h ; b, \mathfrak{T}) P^{*}(s ; b, \mathfrak{T})$ is entire and for $-\infty<c_{1}<c_{2}<\infty$, there is some $c>0$ such that

$$
\left|L(s, h ; b, \mathfrak{T}) P^{*}(s ; b, \mathfrak{T})\right| \ll(|h|(|\Im s|+1))^{c}, \quad c_{1} \leq \Re s \leq c_{2} .
$$

If $m$ is odd and $(-1)^{(m-1) / 2} T h$ is not a perfect square, then already the function $L(s, h ; b, \mathfrak{T}) P^{*}(s ; b, \mathfrak{T})(2 s-m-1)^{-1}$ is entire.

b) The function $L(s, 0 ; b, \mathfrak{T}) P(s ; b, \mathfrak{T})$ is entire and for $-\infty<c_{1}<c_{2}<$ $\infty$, we have

$$
|L(s, 0 ; b, \mathfrak{T}) P(s ; b, \mathfrak{T})| \ll 1
$$

Proof. This is a restatement of Lemmas 3.8 and 3.9 from [5]. The assumption that $\mathfrak{T}$ is positive definite was never used in their proofs. Observe that for $m$ odd and $(-1)^{(m-1) / 2} T h$ not a perfect square, the character $\chi_{(-1)^{(m-1) / 2} 4 \tilde{h}_{\iota} T b^{2}}$ is not the principal character. Here $\tilde{h}_{\iota}$ is defined as in [5], proof of Lemma 3.8. Thus the factor $(s-(m+1) / 2)$ in the equation after (3.17) of [5] is not needed to get an entire function.

The following lemma gives the meromorphic continuation of $\psi$ in certain cases.

Lemma 3.3. Let $\mathfrak{T} \in \mathbb{Z}_{*}^{m \times m}, b \in \mathbb{N}, \alpha \in\{1,2\}, \mu, \nu \in\{0,1\}, q \backslash 2 T b$ a prime and $\chi$ a Dirichlet character modulo $q$. Then

$\psi_{\alpha, \mu, \nu}(\tau, s+\mu ; 1, b, \mathfrak{T}, \chi) P\left(2 s+\mu-\nu+\frac{m}{2}-1 ; b, \mathfrak{T}\right) \Gamma(2 s-\max \{0, \nu-\mu\}-1)^{-1}$

is continuous on $\mathbb{R}^{+} \times \mathbb{C}$ and entire in $s$ for $\tau>0$ fixed.

Proof. The following method is borrowed from the theory of real analytic Eisenstein series. Let $\tau>0, \Re s>m / 4+3$. Dividing the $h$-summation into complete residue systems modulo $k b q$ gives

$$
\begin{aligned}
& \psi_{\alpha, \mu, \nu}(\tau, s+\mu ; 1, b, \mathfrak{T}, \chi) \\
& =(b q)^{-\mu-2 s} \sum_{k \geq 1} \frac{1}{k^{2 s+\mu-\nu+m / 2}} \sum_{1 \leq a \leq b q k} G_{\alpha}(a, b k ; \mathfrak{T}, \chi) \\
& \quad \times \sum_{l \in \mathbb{Z}} \frac{\left(a(b q k)^{-1}+l\right)^{\mu}}{\left(\left(a(b q k)^{-1}+l\right)^{2}+\tau(b q)^{-2}\right)^{s+\mu}}-\tau^{-s-\mu} 0^{\mu} \sum_{k \geq 1} \frac{G_{\alpha}(0, b k ; \mathfrak{T}, \chi)}{k^{2 s+2 \mu-\nu+m / 2}} .
\end{aligned}
$$


Now the $l$-sum is transformed with Poisson's summation formula (see [5], Lemma 3.4, which is a reformulation of a result of [8]). This gives

$$
\begin{aligned}
& \psi=(b q)^{-\mu-2 s}(2 \pi)^{2 s} \Gamma(s)^{-2}\left(\frac{-\pi i}{s}\right)^{\mu} \\
& \times \sum_{l \neq 0} l^{\mu}|l|^{2 s-1} \sigma\left(\frac{4 \pi|l|}{b q} \tau^{1 / 2}, s, s\right) e^{-2 \pi|l| \tau^{1 / 2} /(b q)} \\
& \times \sum_{k \geq 1} \frac{1}{k^{2 s+\mu-\nu+m / 2}} \sum_{1 \leq a \leq b q k} G_{\alpha}(a, b k ; \mathfrak{T}, \chi) e\left(\frac{a l}{b q k}\right) \\
&+(1-\mu)(b q)^{-2 s} \pi 2^{2-2 s} \Gamma(2 s-1) \Gamma(s)^{-2}\left(\frac{\tau}{(b q)^{2}}\right)^{1 / 2-s} \sum_{k \geq 1} \frac{1}{k^{2 s-\nu+m / 2}} \\
& \times \sum_{1 \leq a \leq b q k} G_{\alpha}(a, b k ; \mathfrak{T}, \chi) \\
&-(1-\mu) \tau^{-s} \sum_{k \geq 1} \frac{G_{\alpha}(0, b k ; \mathfrak{T}, \chi)}{k^{2 s-\nu+m / 2}}
\end{aligned}
$$

where $\sigma$ is the confluent hypergeometric function. Since

$$
\sum_{1 \leq a \leq b q k} G_{\alpha}(a, b k ; \mathfrak{T}, \chi) e\left(\frac{a l}{b q k}\right)=b k \lambda(-l, \bar{\chi}) A\left((-1)^{\alpha-1} l, b k ; \mathfrak{T}\right)
$$

and

$$
G_{\alpha}(0, b k ; \mathfrak{T}, \chi)=\left(\frac{b k}{q}\right)^{m} \sum_{c \bmod q} \chi(c) G\left((-1)^{\alpha-1} c, q ; \mathfrak{T}\right)
$$

if $q \mid k$ and $G_{\alpha}(0, b k ; \mathfrak{T}, \chi)=0$ otherwise, it follows that

$$
\begin{aligned}
\psi= & b(b q)^{-\mu-2 s}(2 \pi)^{2 s} \Gamma(s)^{-2}\left(\frac{-\pi i}{s}\right)^{\mu} \sum_{l \neq 0} l^{\mu}|l|^{2 s-1} \sigma\left(\frac{4 \pi|l|}{b q} \tau^{1 / 2}, s, s\right) \\
& \times \lambda(-l, \bar{\chi}) L\left(2 s+\mu-\nu+\frac{m}{2}-1,(-1)^{\alpha-1} l ; b, \mathfrak{T}\right) e^{-2 \pi|l| \tau^{1 / 2} /(b q)} \\
+ & (1-\mu) b(b q)^{-2 s} \pi 2^{2-2 s} \Gamma(2 s-1) \Gamma(s)^{-2}\left(\frac{\tau}{(b q)^{2}}\right)^{1 / 2-s} \lambda(0, \bar{\chi}) \\
& \times L\left(2 s-\nu+\frac{m}{2}-1,0 ; b, \mathfrak{T}\right) \\
& -(1-\mu) \tau^{-s} b^{m} q^{\nu-m / 2-2 s} \zeta\left(2 s-\nu-\frac{m}{2}\right) \sum_{c \bmod q} \chi(c) G\left((-1)^{\alpha-1} c, q ; \mathfrak{T}\right) .
\end{aligned}
$$

Multiplying both sides with $P(2 s+\mu-\nu+m / 2-1 ; b, \mathfrak{T}) \Gamma(2 s-\max \{0, \nu-$ $\mu\}-1)^{-1}$ and using Lemma 3.2 finishes the proof. 
Now the meromorphic continuation of $\phi$ can be proved.

Lemma 3.4. Let $\mathfrak{T} \in \mathbb{Z}_{*}^{m \times m}, T:=\operatorname{det} \mathfrak{T}, \alpha \in\{1,2\}$ and $\mu, \nu \in\{0,1\}$ such that $2(\mu+\nu)+(-1)^{\alpha+1} \operatorname{sign}(\mathfrak{T}) \not \equiv 0 \bmod 4$. Let $q \backslash 2 T$ be prime and $\chi$ a Dirichlet character modulo $q$. Set $b:=1$, $\mathfrak{S}:=\mathfrak{T}$ or $b:=4|T|$, $\mathfrak{S}:=|T| \mathfrak{T}^{-1}$. Then

$$
\phi_{\alpha, \mu, \nu}(\tau, s ; b, \mathfrak{S}, \chi) P\left(2 s-\mu-\nu+\frac{m}{2}-1 ; 1, \mathfrak{T}\right) \Gamma(2 s-2 \max \{\mu, \nu\}-1)^{-1}
$$

is continuous on $\mathbb{R}^{+} \times \mathbb{C}$ and entire in $s$ for fixed $\tau>0$.

Proof. Notice that by the assumption the prefactor of $\phi$ in Lemma 3.1 does not vanish. Therefore Lemmas 3.1 and 3.3 give the statement in case $b=1, \mathfrak{S}=\mathfrak{T}$. On the other hand, the reciprocity law in Proposition 2.3 shows that for $\Re s>m / 4+3, \tau>0$, we have

$$
\begin{aligned}
& \phi_{\alpha, \mu, \nu}(\tau, s ; 1, \mathfrak{T}, \chi) \\
& =2^{-3 m / 2}|T|^{1 / 2-m} e^{\pi i(-1)^{\alpha} \operatorname{sign}(\mathfrak{T}) / 4} C(\chi, \alpha, \mathfrak{T}) \tau^{-s} \\
& \quad \times \phi_{\alpha-1, \nu, \mu}\left(\tau^{-1}, s ; 4|T|,|T| \mathfrak{T}^{-1}, \bar{\chi}\left(\frac{\dot{q}}{q}\right)^{m}\right) .
\end{aligned}
$$

With this identity the statement for $b=4|T|, \mathfrak{S}=|T| \mathfrak{T}^{-1}$ can be deduced from that for $b=1, \mathfrak{S}=\mathfrak{T}$.

The following lemma gives an asymptotic development for $\phi$. It is very similar to the one given for $\psi$ in the proof of Lemma 3.3, with one exception. Since the exponentially decreasing factor is missing in the $l$-sum one cannot derive the meromorphic continuation of $\phi$ from this development. Therefore the continuation of $\phi$ was first proved by using $\psi$. Once this is known, the meromorphic continuation of the terms in the asymptotic development of $\phi$ can be deduced.

Lemma 3.5. Let $\mathfrak{T} \in \mathbb{Z}_{*}^{m \times m}, T:=\operatorname{det} \mathfrak{T}, \alpha \in\{1,2\}$ and $\mu, \nu \in\{0,1\}$ such that $2(\mu+\nu)+(-1)^{\alpha+1} \operatorname{sign}(\mathfrak{T}) \not \equiv 0 \bmod 4$. Let $q \backslash 2 T$ be a prime and $\chi$ a Dirichlet character modulo $q$. Set $b:=1, \mathfrak{S}:=\mathfrak{T}$ or $b:=4|T|$, $\mathfrak{S}:=|T| \mathfrak{T}^{-1}$. There are entire functions

$$
D_{\alpha, \nu}^{*}(s ; b, \mathfrak{T}, \mathfrak{S}, \chi), D_{\mu, \nu}^{* *}(s ; b, \mathfrak{T}, \mathfrak{S}), D_{\alpha, \mu, \nu, \rho}(s ; b, \mathfrak{T}, \mathfrak{S}, \chi), \rho \geq 0,
$$

with the property: For every $\sigma_{1} \in \mathbb{R}^{-}$there is some $r\left(\sigma_{1}\right) \in \mathbb{N}$ such that 


$$
\begin{aligned}
& R_{\alpha, \mu, \nu, r}(\tau, s ; b, \mathfrak{T}, \mathfrak{S}, \chi) \\
& :=\phi_{\alpha, \mu, \nu}(\tau, s ; b, \mathfrak{S}, \chi) P\left(2 s-\mu-\nu+\frac{m}{2}-1 ; 1, \mathfrak{T}\right) \\
& \times \Gamma(2 s-2 \max \{\mu, \nu\}-1)^{-1} \\
& \quad-\sum_{\rho=\mu}^{r-1} \tau^{\mu-s-\rho} D_{\alpha, \mu, \nu, \rho}(s ; b, \mathfrak{T}, \mathfrak{S}, \chi) \\
& -\tau^{\mu-s} D_{\alpha, \nu}^{*}(s ; b, \mathfrak{T}, \mathfrak{S}, \chi)(1-\mu) \\
& -\tau^{(1+\mu) / 2-s} D_{\mu, \nu}^{* *}(s ; b, \mathfrak{T}, \mathfrak{S}) \lambda(0, \bar{\chi})
\end{aligned}
$$

is continuous on $\mathbb{R}^{+} \times \mathbb{C}$ and entire in $s$ for fixed $\tau>0$. For $\Re s \geq \sigma_{1}$, $|s| \leq K, \tau>0$ and $r \geq r\left(\sigma_{1}\right)$, we have

$$
R_{\alpha, \mu, \nu, r}(\tau, s ; b, \mathfrak{S}, \mathfrak{T}, \chi) \ll_{r, K, \sigma_{1}} \tau^{-r+1-\Re s+\mu} .
$$

Proof. Again the $h$-summation is divided into complete residue systems modulo $k b q$ and Poisson's summation formula is applied (see [5], Lemma 3.1). This gives for $\Re s>m / 4+3, \tau>0$, the identity

$$
\begin{aligned}
\phi_{\alpha, \mu, \nu}(\tau, s+\mu ; b, \mathfrak{S}, \chi) & \\
= & b(b q)^{-2 s-\mu} \sum_{k \geq 1} \frac{1}{k^{2 s+\mu-\nu+m / 2-1}} \\
& \times \sum_{l \in \mathbb{Z}} \lambda(l, \bar{\chi}) h_{\mu}\left(l, s, \frac{\tau}{(b q)^{2}}\right) A\left((-1)^{\alpha} l, b k ; \mathfrak{S}\right) \\
- & \frac{1-\mu}{2} b^{m} q^{-2 s+\nu-m / 2} \tau^{-s} \zeta\left(2 s-\nu-\frac{m}{2}\right) \\
& \times \sum_{c \bmod q} \chi(c) G\left((-1)^{\alpha-1} c, q ; \mathfrak{S}\right),
\end{aligned}
$$

where $h_{\mu}(l, s, \tau)$ is the one-sided exponential Fourier transform of $x^{\mu}(\tau+$ $\left.x^{2}\right)^{-s-\mu}$. Its asymptotic behaviour is given in Lemmas 3.2 and 3.3 of [5]. If terms for $l$ and $l-\left[l(b q k)^{-1}\right] 2 b q k$ are grouped together, the $l$-sum becomes absolutely convergent and the value of this absolute sum is $O\left(k^{m+1}\right)$. Plugging in the asymptotic representation for $h_{\mu}(l, s, \tau)$ gives for the $l$-sum the expression

$$
\lambda(0, \bar{\chi}) h_{\mu}\left(0, s, \frac{\tau}{(b q)^{2}}\right) A(0, b k ; \mathfrak{S})
$$




$$
\begin{aligned}
& +s^{-\mu}\left(\frac{\tau}{(b q)^{2}}\right)^{-s} \sum_{\rho=\mu}^{r-1} \tau^{-\rho} p_{\rho}(s) c_{\alpha, \mu, \rho}(k, b ; \mathfrak{S}, \chi) \\
& +s^{-\mu} \sum_{l \neq 0} \lambda(l, \bar{\chi}) A\left((-1)^{\alpha} l, b k ; \mathfrak{S}\right) R_{\mu, r}^{*}\left(l, s ; \frac{\tau}{(b q)^{2}}\right),
\end{aligned}
$$

where

$$
\begin{aligned}
& c_{\alpha, \mu, \rho}(k, b ; \mathfrak{S}, \chi) \ll_{\mu, b, q, \rho} k^{m+1}, \quad p_{\rho}(s) \in \mathbb{Q}[s], \\
& R_{\mu, r}^{*} \ll_{r, q, \mu}|l|^{-2 r+1+\mu} \tau^{-r+1-\Re s}
\end{aligned}
$$

if $r \in \mathbb{N} \backslash\{1\}, \Re s \geq 2-r, \tau>0,|s| \leq K$. Define

$$
\begin{aligned}
& D_{\alpha, \nu}^{*}(s+\mu ; b, \mathfrak{T}, \mathfrak{S}, \chi):=-\frac{1}{2} b^{m} q^{-2 s+\nu-m / 2} \sum_{c \bmod q} \chi(c) G\left((-1)^{\alpha-1} c, q ; \mathfrak{S}\right) \\
& \quad \times \zeta\left(2 s-\nu-\frac{m}{2}\right) P\left(2 s-\nu+\frac{m}{2}-1 ; 1, \mathfrak{T}\right) \Gamma(2 s-2 \nu-1)^{-1}, \\
& D_{\mu, \nu}^{* *}(s+\mu ; b, \mathfrak{T}, \mathfrak{S}) \\
& :=q^{-1} L\left(2 s+\mu-\nu+\frac{m}{2}-1,0 ; b, \mathfrak{S}\right) P\left(2 s+\mu-\nu+\frac{m}{2}-1 ; 1, \mathfrak{T}\right) \\
& \times \Gamma(2 s-2 \max \{0, \nu-\mu\}-1)^{-1}\left\{\begin{array}{c}
\pi 2^{1-2 s} \Gamma(2 s-1) \Gamma(s)^{-2}, \mu=0 \\
(2 s)^{-1}, \mu=1
\end{array}\right\}, \\
& D_{\alpha, \mu, \nu, \rho}(s+\mu ; b, \mathfrak{T}, \mathfrak{S}, \chi):=b(b q)^{-\mu} s^{-\mu} p_{\rho}(s) \sum_{k \geq 1} \frac{c_{\alpha, \mu, \rho}(k, b ; \mathfrak{S}, \chi)}{k^{2 s+\mu-\nu+m / 2-1}} \\
& \times P\left(2 s+\mu-\nu+\frac{m}{2}-1 ; 1, \mathfrak{T}\right) \Gamma(2 s-2 \max \{0, \nu-\mu\}-1)^{-1} .
\end{aligned}
$$

Then $D^{*}$ and $D^{* *}$ are entire and, if $r \geq r\left(\sigma_{1}\right)$,

$$
\begin{aligned}
R_{\alpha, \mu, \nu, r}( & \tau, s+\mu ; b, \mathfrak{T}, \mathfrak{S}, \chi) \\
= & b(b q)^{-2 s-\mu} \sum_{l \neq 0} \lambda(l, \bar{\chi}) R_{\mu, r}^{*}\left(l, s ; \frac{\tau}{(b q)^{2}}\right) s^{-\mu} \\
& \times L\left(2 s+\mu-\nu+\frac{m}{2}-1,(-1)^{\alpha} l ; b, \mathfrak{S}\right) \\
& \times P\left(2 s+\mu-\nu+\frac{m}{2}-1 ; 1, \mathfrak{T}\right) \Gamma(2 s-2 \max \{0, \nu-\mu\}-1)^{-1}
\end{aligned}
$$


is continuous for $\Re s>\sigma_{1}, \tau>0$ and holomorphic in $s$ for $\tau$ fixed. If $|s| \leq K, \Re s>\sigma_{1}$, the value of this function is $O_{r, K, \sigma_{1}}\left(\tau^{-r+1-\Re s}\right)$. Finally, since $D^{*}, D^{* *}$ and the term in $R$ which contains $\phi$ as a factor are entire by Lemma 3.4, it follows that for fixed $\tau>0$, the function

$$
\sum_{\rho=\mu}^{r-1} \tau^{-s-\rho} D_{\alpha, \mu, \nu, \rho}(s+\mu ; b, \mathfrak{T}, \mathfrak{S}, \chi)
$$

is holomorphic on $\Re s>\sigma_{1}$. Choosing $r-\mu$ different values for $\tau$ and solving the corresponding linear system for the functions $D$ shows that $D_{\alpha, \mu, \nu, \rho}(s+\mu ; b, \mathfrak{T}, \mathfrak{S}, \chi), \rho \leq \mu \leq r-1$, are holomorphic on $\Re s>\sigma_{1}$. Since $\sigma_{1} \in \mathbb{R}^{-}$is arbitrary the functions $D$ are in fact entire.

Now the functions $\rho$ can be continued meromorphically.

Theorem 3.6. Let $\mathfrak{T} \in \mathbb{Z}_{*}^{m \times m}, T:=\operatorname{det} \mathfrak{T}, q \backslash 2 T$ prime, $\chi a$ Dirichlet character modulo $q, \alpha \in\{0,1\}$ and $\beta \in\{0,1\}$ such that $2 \beta \not \equiv$ $\operatorname{sign}(\mathfrak{T}) \bmod 4$. Let $a(\chi)=1$ for $\chi=\chi_{0}$ and $a(\chi)=0$ otherwise. Set $b:=1, \mathfrak{S}:=\mathfrak{T}$ or $b:=4|T|, \mathfrak{S}:=|T| \mathfrak{T}^{-1}$. Then

$$
\begin{aligned}
& \rho_{\alpha}(s, w ; b, \mathfrak{S}, \chi) \Gamma\left(\frac{1}{2}\left(s+w+\beta-\frac{m}{2}\right)\right)^{-1} \Gamma\left(s+w-\beta-\frac{m}{2}-1\right)^{-1} \\
& \quad \times P(s+w-1 ; 1, \mathfrak{T})(w-1)^{a(\chi)}\left(s-1-\frac{m}{2}\right)^{a\left(\chi(\dot{\bar{q}})^{m}\right)}
\end{aligned}
$$

is entire in $s$ and $w$ and $O\left((|\Im w|+1)^{c} e^{\pi|\Im w| / 2}\right)$ on every set of the form

$$
\left\{(s, w) \in \mathbb{C}^{2}\left|c_{1} \leq \Re s, \Re w \leq c_{2},\right| s+w \mid \leq c_{3}\right\},
$$

where $c_{1}, c_{2}, c_{3}$ and $c=c\left(c_{1}, c_{2}, c_{3}\right)$ are constants. Furthermore, we have the functional equation

$$
\begin{aligned}
\rho_{\alpha}(s, w ; 1, \mathfrak{T}, \chi)= & 2^{-3 m / 2}|T|^{1 / 2-m} e^{\pi i(-1)^{\alpha} \operatorname{sign}(\mathfrak{T}) / 4} C(\chi, \alpha, \mathfrak{T}) \\
& \times \rho_{\alpha-1}\left(w+\frac{m}{2}, s-\frac{m}{2} ; 4|T|,|T| \mathfrak{T}^{-1}, \bar{\chi}\left(\frac{\dot{q}}{q}\right)^{m}\right) .
\end{aligned}
$$

Proof. Choose $\mu, \nu \in\{0,1\}$ such that $\mu+\nu=\beta$. From (3.1), (3.2) and Lemma 3.5 it follows that, for $\Re s>m+5, \Re w>1$ and $s^{*}:=(s+w+\mu+$ $\nu) / 2-m / 4$, we have

$$
\rho_{\alpha}(s, w ; 1, \mathfrak{T}, \chi) \Gamma\left(\frac{1}{2}\left(s+w+\beta-\frac{m}{2}\right)\right)^{-1} \Gamma\left(s+w-\beta-\frac{m}{2}-1\right)^{-1}
$$




$$
\begin{aligned}
& \times P(s+w-1 ; 1, \mathfrak{T})(w-1)^{a(\chi)}\left(s-1-\frac{m}{2}\right)^{a\left(\chi(\dot{\bar{q}})^{m}\right)}= \\
& \Gamma\left(\frac{s+\nu}{2}-\frac{m}{4}\right)^{-1} \Gamma\left(\frac{w+\mu}{2}\right)^{-1}(w-1)^{a(\chi)}\left(s-1-\frac{m}{2}\right)^{a\left(\chi(\dot{\bar{q}})^{m}\right)} \\
& \times\left\{\left[\int_{1}^{\infty} \tau^{(s+\nu) / 2-m / 4-1} R_{\alpha, \mu, \nu, r}\left(\tau, s^{*} ; 1, \mathfrak{T}, \mathfrak{T}, \chi\right) d \tau\right.\right. \\
& \quad+\sum_{\rho=\mu}^{r-1} \frac{-1}{(s+\nu) / 2-m / 4-s^{*}-\rho+\mu} D_{\alpha, \mu, \nu, \rho}\left(s^{*} ; 1, \mathfrak{T}, \mathfrak{T}, \chi\right) \\
& \quad+\frac{-1}{(s+\nu) / 2-m / 4-s^{*}+\mu} D_{\alpha, \nu}^{*}\left(s^{*} ; 1, \mathfrak{T}, \mathfrak{T}, \chi\right)(1-\mu) \\
& \left.\quad+\frac{-1}{(s+\nu) / 2-m / 4+(1-\mu) / 2-s^{*}+\mu} D_{\mu, \nu}^{* *}\left(s^{*} ; 1, \mathfrak{T}, \mathfrak{T}\right) \lambda(0, \bar{\chi})\right] \\
& +\left[\int_{1}^{-3 m / 2}|T|^{1 / 2-m} e^{\pi i(-1)^{\alpha} \operatorname{sign}(\mathfrak{T}) / 4} C(\chi, \alpha, \mathfrak{T})\right. \\
& +\sum_{\rho=\nu}^{-(s+\nu) / 2+m / 4-1+s^{*}} R_{\alpha-1, \nu, \mu, r}\left(\tau, s^{*} ; 4|T|, \mathfrak{T},|T| \mathfrak{T}-1, \bar{\chi}\left(\frac{\dot{q}}{q}\right)^{m}\right) d \tau \\
& +\frac{-(s+\nu) / 2+m / 4-\rho+\nu}{-(s+\nu) / 2+m / 4+\nu} D_{\alpha-1, \mu}\left(s^{*} ; 4|T|, \mathfrak{T},|T| \mathfrak{T}{ }^{-1}, \bar{\chi}\left(\frac{-}{q}\right)^{m}\right)(1-\nu) \\
& +\frac{-1}{-(s+\nu) / 2+m / 4+(1-\nu) / 2+\nu}\left(s^{*} ; 4|T|, \mathfrak{T},|T| \mathfrak{T}-1, \bar{\chi}\left(\frac{\dot{q}}{q}\right)^{m}\right)
\end{aligned}
$$

The factor in front of the curly brackets cancels the denominators therein. The estimate for $R$ in Lemma 3.5 shows that the integrals define holomorphic functions on

$$
\begin{aligned}
\left\{(s, w) \in \mathbb{C}^{2} \mid\right. & \Re s>2-2 r+\frac{m}{2}+\nu, \\
& \left.\Re w>2-2 r+\mu, \Re s+\Re w>2 \sigma_{1}+\frac{m}{2}-\mu-\nu\right\}
\end{aligned}
$$

if $\sigma_{1} \in \mathbb{R}^{-}$and $r \geq r\left(\sigma_{1}\right)$. Thus the left hand side of (3.5) can be continued holomorphically to the region (3.6). Since $\sigma_{1} \in \mathbb{R}^{-}$is arbitrary the left hand side of (3.5) is entire. Its growth on sets of the form (3.3) can be estimated using Stirling's formula. This establishes the case $b=1, \mathfrak{S}=\mathfrak{T}$. 
The reciprocity law in Proposition 2.3 immediately gives (3.4) for $\Re s>$ $m+2, \Re w>m / 2+2$. From this identity the case $b=4|T|, \mathfrak{S}=|T| \mathfrak{T}^{-1}$ follows easily.

Somewhat more can be deduced from the representation (3.5). The factors $(w-1)^{a(\chi)}$ and $(s-1-m / 2)^{a\left(\chi(\dot{\bar{q}})^{m}\right)}$ are necessary only to cancel the poles of the terms which contain $D_{\mu, \nu}^{* *}$ and $D_{\nu, \mu}^{* *}$ as a factor. This gives

LEMmA 3.7. Under the assumptions of Theorem 3.6 there are entire functions $f_{\beta}(s, w ; b, \mathfrak{S}, \mathfrak{T}, q)$ and $g_{\beta}(s, w ; b, \mathfrak{S}, \mathfrak{T}, q)$ independent of $\alpha$ such that

$$
\begin{aligned}
& \rho_{\alpha}(s, w ; b, \mathfrak{S}, \chi) \Gamma\left(\frac{1}{2}\left(s+w+\beta-\frac{m}{2}\right)\right)^{-1} \\
& \quad \times \Gamma\left(s+w-\beta-\frac{m}{2}-1\right)^{-1} P(s+w-1 ; 1, \mathfrak{T})-\frac{a(\chi)}{w-1} f_{\beta}(s, w ; b, \mathfrak{S}, \mathfrak{T}, q) \\
& \quad-e^{\pi i(-1)^{\alpha} \operatorname{sign}(\mathfrak{T}) / 4} e_{q}^{m(2 \alpha-1)}\left(\frac{T}{q}\right) \frac{a\left(\chi(\dot{\bar{q}})^{m}\right)}{s-1-m / 2} g_{\beta}(s, w ; b, \mathfrak{S}, \mathfrak{T}, q)
\end{aligned}
$$

is entire on $\mathbb{C}^{2}$.

This lemma will be useful in the next section when the number of possible poles of the function $\tau$ is reduced.

\section{§4. Meromorphic continuation of $\tau$}

For $\mathfrak{T} \in \mathbb{Z}_{*}^{m \times m}, T:=\operatorname{det} \mathfrak{T}, \alpha \in\{1,2\}, b \in \mathbb{N}, q \backslash 2 T b$ a prime and $\chi$ a Dirichlet character modulo $q$, define

$$
\tau_{\alpha}(s, w ; b, \mathfrak{T}, \chi):=\sum_{h, k \geq 1} \frac{A\left((-1)^{\alpha-1} h, b k ; \mathfrak{T}\right) \lambda(h, \chi)}{h^{w} k^{s-1}}, \quad \Re s>m+2, \Re w>1 .
$$

Next a connection between $\tau$ and $\rho$ will be established (cf. [5], Section 6). Let $\Re s>m+2, \Re w>1$. Dividing the $h$-summation into complete residue systems modulo $b q k$ gives

$$
\begin{aligned}
& (w-1) \rho_{\alpha}(s, w ; b, \mathfrak{T}, \chi)= \\
& (b q)^{-w}(w-1) \sum_{k \geq 1} \frac{1}{k^{s+w}} \sum_{1 \leq a \leq b q k} G_{\alpha}(a, b k ; \mathfrak{T}, \chi) \zeta\left(w, \frac{a}{b q k}\right),
\end{aligned}
$$


where $\zeta(w, \kappa)$ denotes Hurwitz' zeta function. The right hand side converges uniformly on every compact subset of

$$
U_{R}:=\left\{(s, w) \in \mathbb{C}^{2} \mid \Re s>R+m+4, \Re w>-R\right\} \quad(R \geq 1)
$$

and therefore defines a holomorphic function $F_{R}(s, w)$ on $U_{R}$. On $U_{R} \cap$ $\{\Re w<0\}$ the functional equation of $\zeta(w, \kappa)$ gives

$$
\begin{aligned}
& F_{R}(s, w)=q^{-1}\left(\frac{2 \pi}{b q}\right)^{w-1} \Gamma(1-w)(w-1) \\
& \quad \times\left(e^{i \pi(1-w) / 2} \tau_{\alpha+1}(s+w, 1-w ; b, \mathfrak{T}, \bar{\chi})\right. \\
& \left.\quad+e^{-i \pi(1-w) / 2} \chi(-1) \tau_{\alpha}(s+w, 1-w ; b, \mathfrak{T}, \bar{\chi})\right) .
\end{aligned}
$$

Solving this linear system for $\tau_{\alpha}$ gives

$$
\begin{aligned}
\tau_{\alpha}(s+w, 1-w ; b, \mathfrak{T}, \bar{\chi}) & \\
=\frac{1}{b}\left(\frac{2 \pi}{b q}\right)^{-w} \Gamma(w)\left(e^{i \pi w / 2} \chi(-1) \rho_{\alpha}(s, w ; b, \mathfrak{T}, \chi)\right. & \\
& \left.+e^{-i \pi w / 2} \rho_{\alpha+1}(s, w ; b, \mathfrak{T}, \chi)\right)
\end{aligned}
$$

on $U_{R} \cap\{\Re w<0\}$. Since the $\rho_{\alpha}$ are meromorphic on $\mathbb{C}^{2}$ the same follows for $\tau_{\alpha}$, and consequently (4.1) holds on $\mathbb{C}^{2}$. More precise information is given in

Proposition 4.1. Let $\mathfrak{T} \in \mathbb{Z}_{*}^{m \times m}, T:=\operatorname{det} \mathfrak{T}, q \chi 2 T$ a prime, $\chi a$ Dirichlet character modulo $q, \alpha \in\{1,2\}$ and $\beta \in\{0,1\}$ such that $2 \beta \not \equiv$ $\operatorname{sign}(\mathfrak{T}) \bmod 4$. Set $b:=1, \mathfrak{S}:=\mathfrak{T}$ or $b:=4|T|, \mathfrak{S}:=|T| \mathfrak{T}^{-1}$. Then

$$
\begin{aligned}
(4.2) \tau_{\alpha} & (s, w ; b, \mathfrak{S}, \chi) \Gamma(1-w)^{-1} \Gamma\left(\frac{1}{2}\left(s+\beta-\frac{m}{2}\right)\right)^{-1} \\
& \times \Gamma\left(s-\beta-\frac{m}{2}-1\right)^{-1} P(s-1 ; 1, \mathfrak{T})\left(s+w-2-\frac{m}{2}\right)^{a\left(\chi(\dot{\bar{q}})^{m}\right)}
\end{aligned}
$$

is an entire function of $s$ and $w$. It is bounded by $O\left((|\Im w|+1)^{c} e^{\pi|\Im w|}\right)$ on every set of the form

$$
\left\{(s, w) \in \mathbb{C}^{2}|| s \mid \leq c_{3}, c_{1} \leq \Re w \leq c_{2}\right\}
$$

where $c_{1}, c_{2}, c_{3}, c=c\left(c_{1}, c_{2}, c_{3}\right)$ are constants. 
Proof. The statements follow immediately from (4.1) and Theorem 3.6. Note that by Lemma 3.7 the possible poles of $\rho_{\alpha}$ and $\rho_{\alpha+1}$ at $w=1$ cancel each other.

Next we will reduce the set of possible poles of $\tau$ considerably. This is done with the following simple tools.

LEMMA 4.2. Let $\left(s_{0}, w_{0}\right) \in \mathbb{C}^{2}, U$ an open neighbourhood of this point, $f(s, w)$ holomorphic on $U$ and $f(s, w)\left(s-s_{0}\right)$ entire. Then $f(s, w)$ itself is entire.

Proof. The entire function $F(s, w):=f(s, w)\left(s-s_{0}\right)$ has a Taylor development

$$
F(s, w)=\sum_{k, l \geq 0} a_{k l}\left(s-s_{0}\right)^{k}\left(w-w_{0}\right)^{l}
$$

about $\left(s_{0}, w_{0}\right)$. Since it converges everywhere, $\left|a_{k l}\right| \ll_{R} R^{-k-l}$ for $k, l \geq 0$ and $R>0$. For $w$ close to $w_{0}$, we have $\left(s_{0}, w\right) \in U$ and therefore

$$
\sum_{l \geq 0} a_{0 l}\left(w-w_{0}\right)^{l}=F\left(s_{0}, w\right)=0 .
$$

Thus for $(s, w) \in U, s \neq s_{0}$,

$$
\begin{aligned}
f(s, w) & =\left(s-s_{0}\right)^{-1} F(s, w)=\left(s-s_{0}\right)^{-1} \sum_{k \geq 1, l \geq 0} a_{k l}\left(s-s_{0}\right)^{k}\left(w-w_{0}\right)^{l} \\
& =\sum_{k \geq 1, l \geq 0} a_{k l}\left(s-s_{0}\right)^{k-1}\left(w-w_{0}\right)^{l}
\end{aligned}
$$

Since the right hand power series converges on $\mathbb{C}^{2}$, the function $f$ can be extended to an entire function on $\mathbb{C}^{2}$.

LemmA 4.3. Let $\mathfrak{T} \in \mathbb{Z}_{*}^{m \times m}, T:=\operatorname{det} \mathfrak{T}, b \in \mathbb{N}, q \backslash 2 T b$ a prime, $\chi a$ Dirichlet character modulo $q, k \in \mathbb{N}$ and $\alpha \in\{1,2\}$. Then

$$
\begin{aligned}
& \sum_{h \bmod b q k} A\left((-1)^{\alpha-1} h, b k ; \mathfrak{T}\right) \lambda(h, \chi) \\
& =(b k)^{m} q^{1-m / 2} \cdot \begin{cases}\left(\frac{(-1)^{m / 2} T}{q}\right) \lambda(0, \chi) & , q \mid k, m \text { even }, \\
\epsilon_{q}\left(\frac{(-1)^{(m-1) / 2+\alpha} T}{q}\right)(q-1) & , \quad q \mid k, m \text { odd }, \chi=(\dot{q}), \\
0 & , \text { otherwise. }\end{cases}
\end{aligned}
$$


Proof. Case 1. $q \nmid k$. Then the left hand term in the lemma equals

$$
\sum_{h_{1} \bmod q} \lambda\left(h_{1}, \chi\right) \sum_{h_{2} \bmod b k} A\left((-1)^{\alpha-1} h_{2}, b k ; \mathfrak{T}\right) .
$$

Since the first sum vanishes, the lemma follows.

Case 2. $q \mid k$. Then a simple calculation shows that the left hand term in the lemma equals

$$
(b k)^{m} q^{1-m} \sum_{h \bmod q} \lambda(h, \chi) A\left((-1)^{\alpha-1} h, q ; \mathfrak{T}\right) .
$$

Case 2.1. $m$ even. From [5], Lemma 3.5, it follows that

$$
A\left((-1)^{\alpha-1} h, q ; \mathfrak{T}\right)=q^{m-1}+q^{m / 2-1}\left(\frac{(-1)^{m / 2} T}{q}\right)\left(q \delta_{q}(h)-1\right),
$$

where $\delta_{q}(h)=1$ if $q \mid h$ and $\delta_{q}(h)=0$ otherwise. Thus the sum in (4.3) equals

$$
q^{m / 2}\left(\frac{(-1)^{m / 2} T}{q}\right) \lambda(0, \chi)
$$

and the lemma follows.

Case 2.2. $m$ odd. Then [5], Lemma 3.5, gives

$$
A\left((-1)^{\alpha-1} h, q ; \mathfrak{T}\right)=q^{m-1}+q^{(m-1) / 2}\left(\frac{(-1)^{(m-1) / 2} T(-1)^{\alpha-1} h}{q}\right),
$$

and the sum in (4.3) equals

$$
\begin{aligned}
q^{(m-1) / 2} & \left(\frac{(-1)^{(m-1) / 2+\alpha-1} T}{q}\right) \sum_{h \bmod q} \lambda(h, \chi)\left(\frac{h}{q}\right) \\
& =q^{(m-1) / 2}\left(\frac{(-1)^{(m-1) / 2+\alpha-1} T}{q}\right) q^{1 / 2} \epsilon_{q} \sum_{a \bmod q} \bar{\chi}(-a)\left(\frac{a}{q}\right) .
\end{aligned}
$$

If $\chi=\left(\frac{\dot{q}}{q}\right)$, the last sum equals $\left(\frac{-1}{q}\right)(q-1)$. If $\chi \neq\left(\frac{\dot{q}}{q}\right)$, it equals 0 . Thus the lemma follows.

Define

$\Delta_{\alpha}(s ; b, S, q):=$ 


$$
b^{m-1}(q-1) q^{m / 2-s} \zeta(s-m) \cdot \begin{cases}\left(\frac{(-1)^{m / 2} S}{q}\right), & m \text { even, } \chi=\chi_{0} \\ \epsilon_{q}\left(\frac{(-1)^{(m-1) / 2+\alpha} S}{q}\right), & m \text { odd, } \chi=(\dot{q}) \\ 0 \quad, & \text { otherwise. }\end{cases}
$$

The central result of this section is

TheOREM 4.4. Let $\mathfrak{T} \in \mathbb{Z}_{*}^{m \times m}, T:=\operatorname{det} \mathfrak{T}, q \backslash 2 T$ a prime, $\chi a$ Dirichlet character modulo $q$ and $\alpha \in\{1,2\}$. Set $b:=1, \mathfrak{S}:=\mathfrak{T}$ or $b:=$ $4|T|, \mathfrak{S}:=|T| \mathfrak{T}^{-1}$. Set $S:=\operatorname{det} \mathfrak{S}$. Then

$$
\begin{aligned}
& \left(\tau_{\alpha}(s, w ; b, \mathfrak{S}, \chi)-(w-1)^{-1} \Delta_{\alpha}(s ; b, S, q)\right) \\
& \quad \times P^{*}(s-1 ; 1, \mathfrak{T})\left(s+w-2-\frac{m}{2}\right)^{a\left(\chi(\dot{\bar{q}})^{m}\right)}\left(s-\frac{m}{2}-1\right)^{a\left(\chi(\dot{\bar{q}})^{m}\right)}
\end{aligned}
$$

is entire. Furthermore, we have the functional equation

$$
\begin{aligned}
& \tau_{\alpha}(s, w ; 1, \mathfrak{T}, \chi) \\
& =C(\bar{\chi}, \alpha, \mathfrak{T})\left(\frac{2 \pi}{q}\right)^{s+2 w-2-m / 2}(4|T|)^{2+m / 2-s-w} \chi(-1) 2^{-3 m / 2} \\
& \times|T|^{1 / 2-m} \pi^{-1} \Gamma(1-w) \Gamma\left(2+\frac{m}{2}-s-w\right) \\
& \times\left[\sin \frac{\pi}{2}\left(s+2 w-2-\frac{m}{2}+\frac{1}{2}(-1)^{\alpha+1} \operatorname{sign}(\mathfrak{T})\right)\right. \\
& \times \tau_{\alpha}\left(s, 2+\frac{m}{2}-s-w ; 4|T|,|T| \mathfrak{T}^{-1}, \bar{\chi}\left(\frac{\dot{-}}{q}\right)^{m}\right) \\
& +\chi(-1)\left(\frac{-1}{q}\right)^{m} \sin \frac{\pi}{2}\left(s-\frac{m}{2}+\frac{1}{2}(-1)^{\alpha} \operatorname{sign}(\mathfrak{T})\right) \\
& \left.\times \tau_{\alpha+1}\left(s, 2+\frac{m}{2}-s-w ; 4|T|,|T| \mathfrak{T}^{-1}, \bar{\chi}\left(\frac{\cdot}{q}\right)^{m}\right)\right] .
\end{aligned}
$$

Proof. First we get rid of most of the possible poles. Choose $\beta \in\{0,1\}$ such that $2 \beta \not \equiv \operatorname{sign}(\mathfrak{T}) \bmod 4$. By definition, $\tau_{\alpha}(s, w ; b, \mathfrak{S}, \chi)$ is holomorphic on $U:=\{\Re s>m+2, \Re w>1\}$. Consequently, the function

$$
\begin{gathered}
\tau_{\alpha}(s, w ; b, \mathfrak{S}, \chi)(w-1) \Gamma\left(\frac{1}{2}\left(s+\beta-\frac{m}{2}\right)\right)^{-1} \Gamma\left(s-\beta-\frac{m}{2}-1\right)^{-1} \\
\times P(s-1 ; 1, \mathfrak{T})\left(s+w-2-\frac{m}{2}\right)^{a\left(\chi(\dot{\bar{q}})^{m}\right)}
\end{gathered}
$$

is also holomorphic in this region. According to Proposition 4.1, the function (4.5) becomes entire after multiplication with $(w-1)^{-1} \Gamma(1-w)^{-1}$. 
Lemma 4.2 now shows that in fact (4.5) is already entire. On $U$, we have

$$
\tau_{\alpha}(s, w ; b, \mathfrak{S}, \chi)=\sum_{h \geq 1} \frac{\lambda(h, \chi)}{h^{w}} L\left(s-1,(-1)^{\alpha-1} h ; b, \mathfrak{S}\right)
$$

Let $\sigma_{1} \in \mathbb{R}^{-}$. Lemma 3.2 shows that there is some $c=c\left(\sigma_{1}\right)>0$ such that after multiplication by $P^{*}(s-1 ; 1, \mathfrak{T})$, the series $(4.6)$ converges uniformly on every compact subset of $U\left(\sigma_{1}\right):=\left\{\Re s>\sigma_{1}+1, \Re w>c\left(\sigma_{1}\right)+1\right\}$. Thus the function

$$
\tau_{\alpha}(s, w ; b, \mathfrak{S}, \chi) P^{*}(s-1 ; 1, \mathfrak{T})\left(s+w-2-\frac{m}{2}\right)^{a\left(\chi(\dot{\bar{q}})^{m}\right)}(w-1)
$$

is holomorphic on $U\left(\sigma_{1}\right)$ and, after multiplication with the entire function

$$
\Gamma\left(\frac{1}{2}\left(s+\beta-\frac{m}{2}\right)\right)^{-1} \Gamma\left(s-\beta-\frac{m}{2}-1\right)^{-1} P(s-1 ; 1, \mathfrak{T}) P^{*}(s-1 ; 1, \mathfrak{T})^{-1},
$$

it becomes entire itself (see (4.5)). Again by Lemma 4.2 it follows that (4.7) is already entire.

For $0<\kappa \leq 1$, the function $h(w, \kappa):=\zeta(w, \kappa)-(w-1)^{-1}$ is entire in $w$. Expanding $\zeta(w, \kappa)-\zeta(w)$ into a Dirichlet series for $\Re w>0$ shows that $|h(w, \kappa)| \ll \kappa^{-3 / 2}$ for $0<\kappa \leq 1,|w-1| \leq 1 / 2$. Thus the double series

$$
H(s, w):=\sum_{k \geq 1} \frac{1}{k^{s+w-1}} \sum_{1 \leq a \leq b k q} A\left((-1)^{\alpha-1} a, b k ; \mathfrak{S}\right) \lambda(a, \chi) h\left(w, \frac{a}{b k q}\right)
$$

converges uniformly on every compact subset of $V:=\{\Re s+\Re w>9 / 2+$ $m,|w-1|<1 / 2\}$ and therefore defines a holomorphic function on $V$. On $V^{\prime}:=\{\Re s>9 / 2+m,|w-1|<1 / 2, \Re w>1\}$, we have

$$
\begin{aligned}
& \Delta(s, w):=\tau_{\alpha}(s, w ; b, \mathfrak{S}, \chi)-(b q)^{-w} H(s, w) \\
& =(w-1)^{-1} b^{m-w} q^{m / 2+2-s-2 w}(q-1) \zeta(s+w-m-1) \\
& \times \begin{cases}\left(\frac{(-1)^{m / 2} S}{q}\right) & , m \text { even, } \chi=\chi_{0}, \\
\epsilon_{q}\left(\frac{(-1)^{(m-1) / 2+\alpha} S}{q}\right) & , m \text { odd, } \chi=\left(\frac{\dot{q}}{q}\right), \\
0 & , \text { otherwise. }\end{cases}
\end{aligned}
$$

Since $\left.(w-1) \Delta(s, w)\right|_{w=1}=\Delta_{\alpha}(s ; b, \mathfrak{S}, q)$, we have

$$
\tau_{\alpha}(s, w ; b, \mathfrak{S}, \chi)-(w-1)^{-1} \Delta_{\alpha}(s ; b, S, q)
$$




$$
=(b q)^{-w} H(s, w)+(w-1)^{-1}\left((w-1) \Delta(s, w)-\Delta_{\alpha}(s ; b, S, q)\right)
$$

on $V^{\prime}$, and the right hand side is even holomorphic on $V$. Thus (4.4) is holomorphic on $V$. Since (4.7) is entire, (4.4) becomes so after multiplication with $(w-1)$ (note that the factor $(s-m / 2-1)^{a\left(\chi(\dot{\bar{q}})^{m}\right)}$ is needed to cancel a pole of $\left.P^{*}(s-1 ; 1, \mathfrak{T})\right)$. Applying Lemma 4.2 again shows that (4.4) is entire.

Finally, the functional equation for $\tau_{\alpha}$ follows from that for $\rho_{\alpha}$ (see Theorem 3.6) by means of (4.1).

Finally, the analogue of Theorem 4.4 for untwisted Dirichlet series is needed. Define

$$
\tau_{\alpha}(s, w ; b, \mathfrak{T}):=\sum_{h, k \geq 1} \frac{A\left((-1)^{\alpha-1} h, b k ; \mathfrak{T}\right)}{h^{w} k^{s-1}}, \quad \Re s>m+2, \Re w>1 .
$$

TheOREM 4.5. Let $\mathfrak{T} \in \mathbb{Z}_{*}^{m \times m}, T:=\operatorname{det} \mathfrak{T}, \alpha \in\{1,2\}$. Set $b:=1$, $\mathfrak{S}:=\mathfrak{T}$ or $b:=4|T|, \mathfrak{S}:=|T| \mathfrak{T}^{-1}$. Then

$$
\begin{aligned}
\left(\tau(s, w ; b, \mathfrak{S})-b^{m-1} \zeta(s-m) \frac{1}{w-1}\right) & \\
& \times P^{*}(s-1 ; 1, \mathfrak{T})\left(s+w-2-\frac{m}{2}\right)\left(s-\frac{m}{2}-1\right)
\end{aligned}
$$

is entire. Furthermore,

$$
\begin{aligned}
\tau_{\alpha}(s, & w 1, \mathfrak{T}) \\
= & (2 \pi)^{s+2 w-2-m / 2}(4|T|)^{2+m / 2-s-w} \Gamma(1-w) \\
& \times \Gamma\left(2+\frac{m}{2}-s-w\right) 2^{-3 m / 2}|T|^{1 / 2-m} \pi^{-1} \\
& \times\left[\sin \frac{\pi}{2}\left(s+2 w-2-\frac{m}{2}+\frac{1}{2}(-1)^{\alpha+1} \operatorname{sign}(\mathfrak{T})\right)\right. \\
& \times \tau_{\alpha}\left(s, 2+\frac{m}{2}-s-w ; 4|T|,|T| \mathfrak{T}^{-1}\right) \\
& \quad+\sin \frac{\pi}{2}\left(s-\frac{m}{2}+\frac{1}{2}(-1)^{\alpha} \operatorname{sign}(\mathfrak{T})\right) \\
& \left.\times \tau_{\alpha+1}\left(s, 2+\frac{m}{2}-s-w ; 4|T|,|T| \mathfrak{T}^{-1}\right)\right] .
\end{aligned}
$$

Proof. From [5], Lemmas 6.1 and 6.2, it follows that

$$
\tau_{\alpha}(s, w ; b, \mathfrak{S}) \Gamma(1-w)^{-1}\left(s+w-\frac{m}{2}-2\right)
$$




$$
\times \Gamma\left(\frac{s}{2}-\frac{m}{4}+\frac{\beta}{2}\right)^{-1} \Gamma\left(s-\frac{m}{2}-\beta-1\right)^{-1} P(s-1 ; 1, \mathfrak{T})
$$

is entire if $2 \beta \not \equiv \operatorname{sign}(\mathfrak{T}) \bmod 4$. Furthermore, Theorem 1.4 in [5] immediately gives the functional equation. Exactly as in the proof of Theorem 4.4 it can be shown that already the function

$$
\tau_{\alpha}(s, w ; b, \mathfrak{S})(w-1)\left(s+w-\frac{m}{2}-2\right) P^{*}(s-1 ; 1, \mathfrak{T})
$$

is entire. The remainder of the theorem is proved as there.

\section{§5. Construction of modular forms}

For $\mathfrak{T} \in \mathbb{Z}_{*}^{m \times m}, T:=\operatorname{det} \mathfrak{T}, b \in \mathbb{N}, \alpha \in\{1,2\}, q \backslash 2 T b$ a prime and $\chi$ a Dirichlet character modulo $q$, define

$$
\begin{aligned}
D_{\alpha}(s, w ; b, \mathfrak{T}, \chi) & :=\sum_{n \geq 1} \frac{1}{n^{w}} \mathcal{S}\left(s,(-1)^{\alpha-1} n ; b, \mathfrak{T}\right) \lambda(n, \chi), \\
D_{\alpha}(s, w ; b, \mathfrak{T}) & :=\sum_{n \geq 1} \frac{1}{n^{w}} \mathcal{S}\left(s,(-1)^{\alpha-1} n ; b, \mathfrak{T}\right), \quad \Re s>m+2, \Re w>1 .
\end{aligned}
$$

For $n \in \mathbb{Z} \backslash\{0\}$ and $\Re s>m+2$, a simple calculation shows that

$$
\zeta(s-m) \mathcal{S}(s, n ; b, \mathfrak{T})=b L(s-1, n ; b, \mathfrak{T}) .
$$

From Lemma 3.2 it follows that $\mathcal{S}(s, n ; b, \mathfrak{T})$ can be continued to a meromorphic function on $\mathbb{C}$. There are no poles to the right of $\sigma(m)$, where $\sigma(m):=(m+2) / 2$ if $m$ is even and $\sigma(m):=(m+3) / 2$ if $m$ is odd. The same lemma shows that there is some $c>0$ such that

$$
D_{\alpha}(s, w ; b, \mathfrak{T}, \chi)=b \tau_{\alpha}(s, w ; b, \mathfrak{T}, \chi) \zeta(s-m)^{-1}
$$

converges uniformly as a Dirichlet series in $w$ on every compact subset of $\{\Re s>\sigma(m), \Re w>c\}$; in particular it defines a holomorphic function there. The same holds true for

$$
D_{\alpha}(s, w ; b, \mathfrak{T})=b \tau_{\alpha}(s, w ; b, \mathfrak{T}) \zeta(s-m)^{-1} .
$$

Proposition 5.1. Let $\mathfrak{T} \in \mathbb{Z}_{*}^{m \times m}, T:=\operatorname{det} \mathfrak{T}, \alpha \in\{1,2\}, q \backslash 2 T$ a prime and $\chi$ a Dirichlet character modulo $q$. Set $b:=1, \mathfrak{S}:=\mathfrak{T}$ or $b:=4|T|, \mathfrak{S}:=|T| \mathfrak{T}^{-1}$. Set $S:=\operatorname{det} \mathfrak{S}$ and let $l \in \mathbb{Z}$ be such that

$$
s_{\alpha l}:=\frac{m}{2}+\frac{1}{2}(-1)^{\alpha-1} \operatorname{sign}(\mathfrak{T})+2 l>\sigma(m) .
$$


Then $D_{\alpha}\left(s_{\alpha l}, w ; b, \mathfrak{S}, \chi\right)$ is meromorphic on $\mathbb{C}$. If $\chi(\dot{\bar{q}})^{m} \neq \chi_{0}$ then it is entire. If $\chi(\dot{q})^{m}=\chi_{0}$ then it has one pole at $w=1$ which is simple and has the residue

$r_{\alpha l}(b, \mathfrak{S}, q):=b^{m}(q-1) q^{m / 2-s_{\alpha l}} \cdot\left\{\begin{array}{ll}\left(\frac{(-1)^{m / 2} S}{q}\right) & m \text { even, } \chi=\chi_{0}, \\ \epsilon_{q}\left(\frac{(-1)^{(m-1) / 2+\alpha} S}{q}\right), & m \text { odd, } \chi=(\dot{q}) .\end{array}\right\}$

Furthermore, $D_{\alpha}\left(s_{\alpha l}, w ; b, \mathfrak{S}\right)$ is meromorphic on $\mathbb{C}$ with a simple pole at $w=1$ with residue $b^{m}$. If $s_{\alpha l} \neq m / 2+2$ then there are no further poles of $D_{\alpha}\left(s_{\alpha l}, w ; b, \mathfrak{S}, \chi\right)$ and $D_{\alpha}\left(s_{\alpha l}, w ; b, \mathfrak{S}\right)$. If $s_{\alpha l}=m / 2+2$ then there may be another simple pole at $w=0$.

We have the functional equations

$$
\begin{aligned}
& D_{\alpha}\left(s_{\alpha l}, w ; 1, \mathfrak{T}, \chi\right) \\
&= C(\bar{\chi}, \alpha, \mathfrak{T}) 2^{-3 m / 2-2}|T|^{-1 / 2-m} \pi^{-1} \chi(-1)\left(\frac{2 \pi}{q}\right)^{s_{\alpha l}+2 w-2-m / 2} \\
& \times(4|T|)^{2+m / 2-s_{\alpha l}-w} \Gamma(1-w) \Gamma\left(2+\frac{m}{2}-s_{\alpha l}-w\right) \\
& \times \sin \frac{\pi}{2}\left(s_{\alpha l}+2 w-2-\frac{m}{2}+\frac{1}{2}(-1)^{\alpha+1} \operatorname{sign}(\mathfrak{T})\right) \\
& \times D_{\alpha}\left(s_{\alpha l}, 2+\frac{m}{2}-s_{\alpha l}-w ; 4|T|,|T| \mathfrak{T}^{-1}, \bar{\chi}\left(\frac{\cdot}{q}\right)^{m}\right)
\end{aligned}
$$

$$
\begin{aligned}
D_{\alpha} & \left(s_{\alpha l}, w ; 1, \mathfrak{T}\right) \\
= & 2^{-3 m / 2-2}|T|^{-1 / 2-m} \pi^{-1}(2 \pi)^{s_{\alpha l}+2 w-2-m / 2}(4|T|)^{2+m / 2-s_{\alpha l}-w} \Gamma(1-w) \\
& \times \Gamma\left(2+\frac{m}{2}-s_{\alpha l}-w\right) \sin \frac{\pi}{2}\left(s_{\alpha l}+2 w-2-\frac{m}{2}+\frac{1}{2}(-1)^{\alpha+1} \operatorname{sign}(\mathfrak{T})\right) \\
& \quad \times D_{\alpha}\left(s_{\alpha l}, 2+\frac{m}{2}-s_{\alpha l}-w ; 4|T|,|T| \mathfrak{T}^{-1}\right) .
\end{aligned}
$$

Finally, on every vertical strip of finite width $\left\{c_{1} \leq \Re w \leq c_{2},|\Im w| \geq\right.$ $1\}$, we have

$$
D_{\alpha}\left(s_{\alpha l}, w ; b, \mathfrak{S}, \chi\right) \ll|\Im w|^{c} e^{\pi|\Im w| / 2}
$$

where $c=c\left(c_{1}, c_{2}\right)>0$ is a constant. The same holds for $D_{\alpha}\left(s_{\alpha l}, w ; b, \mathfrak{S}\right)$.

Proof. We only have to compile the results so far. From (5.1), (5.2) and Theorems 4.4 and 4.5 it follows that

$$
\left(D_{\alpha}(s, w ; b, \mathfrak{S}, \chi)-(w-1)^{-1} b \zeta(s-m)^{-1} \Delta_{\alpha}(s, b ; S, q)\right) \zeta(s-m)
$$




$$
\times P^{*}(s-1 ; 1, \mathfrak{T})\left(s+w-2-\frac{m}{2}\right)^{a\left(\chi(\dot{\bar{q}})^{m}\right)}\left(s-\frac{m}{2}-1\right)^{a\left(\chi(\dot{\bar{q}})^{m}\right)}
$$

and

$$
\begin{gathered}
\left(D_{\alpha}(s, w ; b, \mathfrak{S})-b^{m}(w-1)^{-1}\right) \zeta(s-m) P^{*}(s-1 ; 1, \mathfrak{T}) \\
\times\left(s+w-2-\frac{m}{2}\right)\left(s-\frac{m}{2}-1\right)
\end{gathered}
$$

are entire. Since the factors depending on $s$ have no zero or pole at $s=s_{\alpha l}$ it follows that

$$
\begin{aligned}
\left(D_{\alpha}\left(s_{\alpha l}, w ; b, \mathfrak{S}, \chi\right)-(w-1)^{-1} b \zeta(s\right. & \left.-m)\left.^{-1} \Delta_{\alpha}(s ; b, S, q)\right|_{s=s_{\alpha l}}\right) \\
& \times\left(s_{\alpha l}+w-2-\frac{m}{2}\right)^{a\left(\chi(\dot{q})^{m}\right)}
\end{aligned}
$$

and

$$
\left(D_{\alpha}\left(s_{\alpha l}, w ; b, \mathfrak{S}\right)-b^{m}(w-1)^{-1}\right)\left(s_{\alpha l}+w-2-\frac{m}{2}\right)
$$

are entire. If $\chi\left(\frac{\dot{q}}{q}\right)^{m} \neq \chi_{0}$ then $D_{\alpha}\left(s_{\alpha l}, w ; b, \mathfrak{S}, \chi\right)$ is entire. If $\chi\left(\frac{\dot{q}}{q}\right)^{m}=\chi_{0}$ then

$$
\left(D_{\alpha}\left(s_{\alpha l}, w ; b, \mathfrak{S}, \chi\right)-(w-1)^{-1} r_{\alpha l}(b, \mathfrak{S}, q)\right)\left(s_{\alpha l}+w-2-\frac{m}{2}\right)
$$

is entire. This means that $D_{\alpha}\left(s_{\alpha l}, w ; b, \mathfrak{S}, \chi\right)$ has a simple pole at $w=1$ with residue $r_{\alpha l}(b, \mathfrak{S}, q)$, and at most another pole at $w=2+m / 2-s_{\alpha l}<1$ of order 1 . Similarly, $D_{\alpha}\left(s_{\alpha l}, w ; b, \mathfrak{S}\right)$ has a simple pole at $w=1$ with residue $b^{m}$, and at most another pole at $2+m / 2-s_{\alpha l}$ of order 1 .

The functional equations for $D_{\alpha}$ follow immediately from those in Theorems 4.4 and 4.5 with the help of (5.1) and (5.2) since the second summands on the right hand sides are holomorphic and vanish at $s=s_{\alpha l}$. Now if $D_{\alpha}\left(s_{\alpha l}, w ; b, \mathfrak{S}, \chi\right)$ has a pole at $2+m / 2-s_{\alpha l}$ then $D_{\alpha}\left(s_{\alpha l}, w ; 4|T| b^{-1},|T| \mathfrak{S}^{-1}\right.$, $\left.\bar{\chi}(\dot{q})^{m}\right)$ has a pole at 0 by the functional equation. Thus $2+m / 2-s_{\alpha l}=0$. The same holds for $D_{\alpha}\left(s_{\alpha l}, w ; b, \mathfrak{S}\right)$.

Finally, the growth estimates in vertical strips of finite width follow immediately from those in Proposition 4.1. If in (4.2) the functions depending only on $s$ vanish at $s=s_{\alpha l}$ then divide by a suitable power of $\left(s-s_{\alpha l}\right)$ and integrate along a small circle about $s_{\alpha l}$ with respect to $s$.

Now Theorem 1.1 can be proved by applying Weil's converse theorem. We introduce some standard notation. For a sequence $\left(a_{n}\right)_{n \geq 0}$ in $\mathbb{C}$ with 
$a_{n}=O\left(n^{c}\right)$ as $n \rightarrow \infty$ with a constant $c>0$, set $f(z):=\sum_{n \geq 0} a_{n} e(n z)$, $z \in \mathbb{H}$. For a Dirichlet character $\chi$ modulo $q$, set

$$
L(w ; f, \chi):=\sum_{n \geq 1} \frac{a_{n} \chi(n)}{n^{w}}, \quad L(w ; f):=\sum_{n \geq 1} \frac{a_{n}}{n^{w}} .
$$

For $A>0$, set

$$
\begin{aligned}
& \Lambda_{A}(w ; f, \chi):=\left(\frac{2 \pi}{q A^{1 / 2}}\right)^{-w} \Gamma(w) L(w ; f, \chi) \\
& \Lambda_{A}(w ; f):=\left(\frac{2 \pi}{A^{1 / 2}}\right)^{-w} \Gamma(w) L(w ; f) .
\end{aligned}
$$

Furthermore, define

$$
f(z)_{q}:=q^{1 / 2} \sum_{n \geq 0} a_{q n} e(q n z)-q^{-1 / 2} f(z), \quad f(z)_{\chi}:=\sum_{n \geq 0} a_{n} \chi(n) e(n z) .
$$

Now let the assumptions of Theorem 1.1 be fulfilled. Define $\lambda_{\alpha l}:=$ $\left(\kappa_{\alpha l}-1\right) / 2$. For $q \backslash 2 T$ a prime and $\chi$ a Dirichlet character modulo $q$, we have

$$
\begin{aligned}
& \Lambda_{4|T|}\left(w ; \Theta_{\alpha l}(\cdot ; b, \mathfrak{S}), \chi\right)= \\
& \quad \pi^{-w} q^{w}|T|^{w / 2} \Gamma(w) \overline{\tau(\chi)}^{-1} D_{\alpha}\left(s_{\alpha l}, w+1-\frac{\kappa_{\alpha l}}{2} ; b, \mathfrak{S}, \chi\right)
\end{aligned}
$$

for $\chi \neq \chi_{0}$,

$$
\begin{aligned}
& \Lambda_{4|T| q^{2}}\left(w ; \Theta_{\alpha l}(\cdot ; b, \mathfrak{S})_{q}\right)= \\
& \quad \pi^{-w} q^{w}|T|^{w / 2} \Gamma(w) q^{-1 / 2} D_{\alpha}\left(s_{\alpha l}, w+1-\frac{\kappa_{\alpha l}}{2} ; b, \mathfrak{S}, \chi_{0}\right)
\end{aligned}
$$

for $\chi=\chi_{0}$,

$$
\begin{aligned}
& \Lambda_{4|T| q^{2}}\left(w ; \Theta_{\alpha l}(\cdot ; b, \mathfrak{S})_{(\dot{q})}\right)= \\
& \quad \pi^{-w} q^{w}|T|^{w / 2} \Gamma(w) \overline{\tau\left(\left(\frac{\cdot}{q}\right)\right)}
\end{aligned}
$$

for $\chi=(\dot{q})$, and

$$
\Lambda_{4|T|}\left(w ; \Theta_{\alpha l}(\cdot ; b, \mathfrak{S})\right)=\pi^{-w}|T|^{w / 2} \Gamma(w) D_{\alpha}\left(s_{\alpha l}, w+1-\frac{\kappa_{\alpha l}}{2} ; b, \mathfrak{S}\right) .
$$


It follows from Proposition 5.1 that all these functions are meromorphic on $\mathbb{C}$ and grow at most polynomially in vertical strips of finite width.

Case 1. $m$ odd. Proposition 5.1 shows that for $\chi \neq \chi_{0},(\dot{q})$,

$$
\begin{aligned}
& \Lambda_{4|T|}\left(w ; \Theta_{\alpha l}(\cdot ; 1, \mathfrak{T}), \chi\right)= \\
& \quad C_{\chi}^{(1)} C_{\alpha l} \Lambda_{4|T|}\left(\frac{\kappa_{\alpha l}}{2}-w ; \Theta_{\alpha l}\left(\cdot ; 4|T|,|T| \mathfrak{T}^{-1}\right), \bar{\chi}\left(\frac{\cdot}{q}\right)\right),
\end{aligned}
$$

where

$$
\begin{aligned}
C_{\alpha l} & :=(-1)^{l}|T|^{1 / 2-m-\kappa_{\alpha l} / 4} 2^{-\kappa_{\alpha l} / 2-3 m / 2} \\
C_{\chi}^{(1)} & :=\frac{\tau\left(\chi\left(\frac{\dot{q}}{q}\right)\right)}{\tau(\bar{\chi})} \epsilon_{q}^{-1} \chi(-4|T|)\left(\frac{4|T|}{q}\right)\left(\frac{-1}{q}\right)^{\lambda_{\alpha l}} \chi_{4|T|}(q),
\end{aligned}
$$

and both sides of the equation are entire. For $\chi=(\dot{q})$,

$\Lambda_{4|T| q^{2}}\left(w ; \Theta_{\alpha l}(\cdot ; 1, \mathfrak{T})_{(\dot{q})}\right)=C_{\chi}^{(2)} C_{\alpha l} \Lambda_{4|T| q^{2}}\left(\frac{\kappa_{\alpha l}}{2}-w ; \Theta_{\alpha l}\left(\cdot ; 4|T|,|T| \mathfrak{T}^{-1}\right)_{q}\right)$

where

$$
C_{\chi}^{(2)}:=\left(\frac{-1}{q}\right)^{\lambda_{\alpha l}} \chi_{4|T|}(q) .
$$

Proposition 5.1 and this functional equation show that the left hand side has a single pole at $w=\kappa_{\alpha l} / 2$ which is simple and has the residue

$$
\pi^{-\kappa_{\alpha l} / 2}|T|^{\kappa_{\alpha l} / 4} \Gamma\left(\frac{\kappa_{\alpha l}}{2}\right)\left(q^{1 / 2}-q^{-1 / 2}\right) C_{\chi}^{(2)}
$$

Finally,

$$
\Lambda_{4|T|}\left(w ; \Theta_{\alpha l}(\cdot ; 1, \mathfrak{T})\right)=C_{\alpha l} \Lambda_{4|T|}\left(\frac{\kappa_{\alpha l}}{2}-w ; \Theta_{\alpha l}\left(\cdot ; 4|T|,|T| \mathfrak{T}^{-1}\right)\right) .
$$

Proposition 5.1 and this functional equation show that the left hand side has only poles at $w=\kappa_{\alpha l} / 2$ and $w=0$; both are simple and have residues

$$
\pi^{-\kappa_{\alpha l} / 2}|T|^{\kappa_{\alpha l} / 4} \Gamma\left(\frac{\kappa_{\alpha l}}{2}\right) \text { resp. } \quad-\pi^{-\kappa_{\alpha l} / 2}|T|^{\kappa_{\alpha l} / 4} \Gamma\left(\frac{\kappa_{\alpha l}}{2}\right)(4|T|)^{m} C_{\alpha l} .
$$

Now a version of Weil's converse theorem for modular forms of half integral weight can be applied ([12]; the case of half integral weight was scetched in [6] and worked out in [1]). This gives case a) of Theorem 1.1. 
Case 2. $m$ even. Proposition 5.1 shows that for $\chi \neq \chi_{0}$,

$$
\Lambda_{4|T|}\left(w ; \Theta_{\alpha l}(\cdot ; 1, \mathfrak{T}), \chi\right)=C_{\chi} C_{\alpha l} \Lambda_{4|T|}\left(\frac{\kappa_{\alpha l}}{2}-w ; \Theta_{\alpha l}\left(\cdot ; 4|T|,|T| \mathfrak{T}^{-1}\right), \bar{\chi}\right),
$$

where

$$
C_{\chi}:=\frac{1}{q} \tau(\chi)^{2} \chi(4|T|) \chi_{(-1)^{m / 2} 4 T}(q),
$$

and both sides are entire. Weil's converse theorem now gives case b) of Theorem 1.1.

For a comparison between Theorem 1.1 and Cohen's result [2], Theorem 3.1, set $m=1, \mathfrak{T}=(1)$. Let $r \in \mathbb{N}, r \geq 2$. There is exactly one pair $(\alpha, l) \in\{1,2\} \times \mathbb{Z}$ with $s_{\alpha l}=r+1$. For this pair, we have $\alpha \equiv r+1 \bmod 2$ and $\kappa_{\alpha l} / 2=r+1 / 2$. Furthermore, $s_{\alpha l}>\sigma(1)=2$. In [5], Section 8, it was shown that, for $\Re s, \Re w>1$,

$$
\begin{aligned}
8 \zeta(2(s+w)-1) \sum_{D(-1)^{\alpha-1}>0, D \text { a discr. }} \frac{L\left(s, \chi_{D}\right)}{|D|^{w}} & =4 \frac{\zeta(2 s)}{\zeta(s)} \tau_{\alpha}(s+1, w ; 4,(1)) \\
& =\zeta(2 s) D_{\alpha}(s+1, w ; 4,(1)) .
\end{aligned}
$$

Comparing coefficients with respect to $w$ gives

$$
\begin{aligned}
& \sum_{d \geq 1, D(-1)^{\alpha-1}>0: D \text { a discr., } d^{2}|D|=n} \frac{8}{d^{2 s-1}} L\left(s, \chi_{D}\right)= \\
& \zeta(2 s) \mathcal{S}\left(s+1,(-1)^{\alpha-1} n ; 4,(1)\right)
\end{aligned}
$$

for $n \in \mathbb{N}, s \in \mathbb{C}$ not a pole. Plugging in $s=r$ gives

$$
\begin{aligned}
& \zeta(2 r) \mathcal{S}\left(s_{\alpha l},(-1)^{\alpha-1} n ; 4,(1)\right)= \\
& \pi^{r} 2^{r+2} n^{1 / 2-r} \frac{1}{(r-1) !}(-1)^{[r / 2]} H(r, n), \quad n \in \mathbb{N},
\end{aligned}
$$

where the "generalized Hurwitz class numbers" $H(r, n)$ are defined in [2], Definitions 2.1 and 2.2. Furthermore,

$$
\pi^{-\kappa_{\alpha l} / 2}(-1)^{l} \Gamma\left(\frac{\kappa_{\alpha l}}{2}\right) \zeta(2 r)=\pi^{r} \zeta(1-2 r) \frac{1}{(r-1) !}(-1)^{[r / 2]} .
$$

As in [2], we set $H(r, 0):=\zeta(1-2 r)$. Thus for $z \in \mathbb{H}$,

$$
\mathcal{H}_{r}(z):=\sum_{n \geq 0} H(r, n) e(n z)=\zeta(2 r) \pi^{-r} 2^{-2-r}(r-1) !(-1)^{[r / 2]} \Theta_{\alpha l}(z ; 4,(1)) .
$$

It now follows from Theorem 1.1 that $\mathcal{H}_{r}(z)$ is a modular form of weight $r+1 / 2$ with respect to $\Gamma_{0}(4)$, the theta multiplier system and character $\chi_{0} \bmod 4$. This is Theorem 3.1 in [2]. 


\section{§6. Calculation of a singular component of $\tau$}

From now on let $m$ be odd. In Proposition 5.1 only values $s_{\alpha l}>$ $(m+3) / 2$ were allowed in the first argument of $D_{\alpha}$. This was to ensure that we would not fall into a pole of $\mathcal{S}(s, n ; b, \mathfrak{T})$. This procedure is in fact over-cautious. For one value of $\alpha$, depending on $m$ and the sign of $T$, there is no pole at $(m+3) / 2$. For the other value of $\alpha$ the terms in the series development of $\tau_{\alpha}$ which contribute to the pole at $(m+3) / 2$ can be precisely identified. Thus - excerting some care - the value $(m+3) / 2$ can be plugged in the functional equation of $\tau_{\alpha}$. For one value of $\alpha$ this gives a functional equation for polynomials occuring in the singular component of $\tau_{\alpha}$. For the other value of $\alpha$ it gives a functional equation of $\tau_{\alpha}$ with an additional term.

For $a \in \mathbb{Z} \backslash\{0\}$, let $a^{*}$ denote the squarefree kernel of $a$ including the sign.

Lemma 6.1. Let $m \in \mathbb{N}$ be odd, $\mathfrak{T} \in \mathbb{Z}_{*}^{m \times m}, T:=\operatorname{det} \mathfrak{T}$ and $\alpha \in\{1,2\}$ such that $\alpha \equiv(m+1) / 2+(1-\operatorname{sign}(T)) / 2 \bmod 2$. Let $b \in \mathbb{N}$. For $p \mid 2 T b$ prime, there are polynomials $R_{p}(x, y) \in \mathbb{Q}[x, y]$ depending only on $\mathfrak{T}, b$ and $p$, with the properties:

a) For $q \backslash 2 T b$ a prime and $\chi$ a Dirichlet character modulo $q$, define

$$
\begin{aligned}
& \tau_{\alpha}^{*}(s, w ; b, \mathfrak{T}, \chi):= \\
& \quad \sum_{h \geq 1: h^{*}=\left|T^{*}\right|} \frac{\lambda(h, \chi)}{h^{w}} L\left(s-1,(-1)^{\alpha-1} h ; b, \mathfrak{T}\right), \quad \Re s>m+1, \Re w>1 . \\
& \quad \text { If } \chi \neq \chi_{0} \text { then } \\
& \tau_{\alpha}^{*}(s+1, w ; b, \mathfrak{T}, \chi)=\overline{\tau(\chi)} \chi\left(\left|T^{*}\right|\right) b^{s}\left|T^{*}\right|^{-w} L\left(2 s+2 w-m, \chi^{2}\right) L\left(2 w, \chi^{2}\right) \\
& \quad \times \zeta(s-m+1) \zeta(2 s-m+1)^{-1} \zeta\left(s-\frac{m-1}{2}\right) L\left(s+2 w-\frac{m-1}{2}, \chi^{2}\right)^{-1} \\
& \times \prod_{p \mid 2 T b} \frac{R_{p}\left(p^{-s}, \chi^{2}(p) p^{-2 w}\right)}{\left(1+p^{(m-1) / 2-s}\right)\left(1-\chi^{2}(p) p^{(m-1) / 2-s-2 w)}\right.} . \\
& \quad \text { If } \chi=\chi_{0} \text { then } \\
& \tau_{\alpha}^{*}\left(s+1, w ; b, \mathfrak{T}, \chi_{0}\right)=b^{s}\left|T^{*}\right|^{-w} \zeta(2 s+2 w-m) \zeta(2 w) \\
& \times \zeta(s-m+1) \zeta(2 s-m+1)^{-1} \zeta\left(s-\frac{m-1}{2}\right) \zeta\left(s+2 w-\frac{m-1}{2}\right)^{-1}
\end{aligned}
$$




$$
\begin{aligned}
& \times \prod_{p \mid 2 T b} \frac{R_{p}\left(p^{-s}, p^{-2 w}\right)}{\left(1+p^{(m-1) / 2-s}\right)\left(1-p^{(m-1) / 2-s-2 w}\right)} \\
& \times \frac{q^{1-2 w}+q^{m+1-2 s-2 w}+q^{(m-1) / 2-s-2 w}-q^{(m+1) / 2-s-2 w}-q^{m+1-2 s-4 w}-1}{1-q^{(m-1) / 2-s-2 w}} .
\end{aligned}
$$

b) If $\chi$ is the principal character modulo 1 , define

$$
\begin{aligned}
& \tau_{\alpha}^{*}(s, w ; b, \mathfrak{T}):= \\
& \quad \sum_{h \geq 1: h^{*}=\left|T^{*}\right|} \frac{1}{h^{w}} L\left(s-1,(-1)^{\alpha-1} h ; b, \mathfrak{T}\right), \quad \Re s>m+1, \Re w>1 .
\end{aligned}
$$

Then

$$
\begin{aligned}
& \tau_{\alpha}^{*}(s+1, w ; b, \mathfrak{T})=b^{s}\left|T^{*}\right|^{-w} \zeta(2 s+2 w-m) \zeta(2 w) \\
& \quad \times \zeta(s-m+1) \zeta(2 s-m+1)^{-1} \zeta\left(s-\frac{m-1}{2}\right) \zeta\left(s+2 w-\frac{m-1}{2}\right)^{-1} \\
& \quad \times \prod_{p \mid 2 T b} \frac{R_{p}\left(p^{-s}, p^{-2 w}\right)}{\left(1+p^{(m-1) / 2-s}\right)\left(1-p^{(m-1) / 2-s-2 w}\right)}
\end{aligned}
$$

Proof. Let $r \in \mathbb{N}, e \in \mathbb{N}_{0}$ and $\psi$ a Dirichlet character modulo $r$. Define $f\left(s, w ; q^{e}, \psi\right):=\sum_{n \geq 1} \frac{\psi(n)}{n^{w}} L\left(s,(-1)^{\alpha-1}\left|T^{*}\right| n^{2} q^{2 e} ; b, \mathfrak{T}\right), \Re s>m+1, \Re w>1$.

Let $b=\prod_{p} p^{a_{p}}$ be the prime factorisation of $b$. The Chinese Remainder Theorem shows that

$$
b^{-s} L\left(s,(-1)^{\alpha-1}\left|T^{*}\right| n^{2} q^{2 e} ; b, \mathfrak{T}\right)=\prod_{p} L_{p}\left(s, n q^{e}\right), \quad \Re s>m+1,
$$

where

$$
L_{p}(s, n):=\sum_{a \geq a_{p}} p^{-a s} A\left((-1)^{\alpha-1}\left|T^{*}\right| n^{2}, p^{a} ; \mathfrak{T}\right) .
$$

Let $p^{l_{p}} \| n^{2}$. Then $A\left((-1)^{\alpha-1}\left|T^{*}\right| n^{2} q^{2 e}, p^{a} ; \mathfrak{T}\right)=A\left((-1)^{\alpha-1}\left|T^{*}\right| p^{2 l_{p}} q^{2 e}, p^{a} ; \mathfrak{T}\right)$. If $q \neq p$ the factor $q^{2 e}$ on the right hand side may be omitted. Thus

$$
b^{-s} f\left(s, w ; q^{e}, \psi\right)=\prod_{p}\left(\sum_{l_{p} \geq 0} \frac{\psi\left(p^{l_{p}}\right)}{p^{l_{p} w}} L_{p}\left(s, p^{l_{p}} q^{e \delta_{p q}}\right)\right)
$$


for $\Re s, \Re w$ sufficiently large.

Let $p \nmid 2 T b$. Since $\alpha$ has been chosen appropriately, (3.16) in [5] gives

$$
\begin{aligned}
& L_{p}\left(s, p^{l}\right)\left(1-p^{m-1-s}\right)\left(1-p^{m-1-2 s}\right)^{-1} \\
& \quad=1+p^{m-2 s}+\cdots+p^{(m-2 s)(l-1)}+p^{(m-2 s) l}\left(1-p^{(m-1) / 2-s}\right)^{-1} .
\end{aligned}
$$

Thus for $\Re s>m+1, \Re w>1$, we have

$$
\begin{aligned}
& \sum_{l_{p} \geq 0} \frac{\psi\left(p^{l_{p}}\right)}{p^{l_{p} w}} L_{p}\left(s, p^{l_{p}}\right)= \\
& \quad \frac{\left(1-p^{m-1-2 s}\right)\left(1-\psi(p) p^{(m-1) / 2-s-w}\right)}{\left(1-p^{m-1-s}\right)\left(1-\psi(p) p^{m-2 s-w}\right)\left(1-p^{(m-1) / 2-s}\right)\left(1-\psi(p) p^{-w}\right)}
\end{aligned}
$$

Our goal now is to show that for $p \mid 2 T b$ the left hand side is still a rational function in $p^{-s}$ and $\psi(p) p^{-w}$. The same proof as for (3.13) in [5] works for the following more general statement: There are $\kappa \in \mathbb{Q}$ and $a_{p}^{*} \in \mathbb{N}$ such that for $c \in \mathbb{N}_{0}, a \geq a_{p}^{*}$ and $d \in \mathbb{Z} \backslash\{0\}$, we have

$$
A\left(d p^{c}, p^{a} ; \mathfrak{T}\right)=\kappa(c, d) p^{(m-1) a}+A\left(d p^{c-2}, p^{a-2} ; \mathfrak{T}\right) p^{m},
$$

where $\kappa(c, d) \in \mathbb{Q}$ depends only on $c$ and $d$, and $\kappa(c, d)=\kappa$ for $c \geq a_{p}^{*}$. Denote the left hand side of $(6.3)$ by $f_{p}(s, w ; \psi)$. Then for $\Re s>m+1$, $\Re w>1$, we have

$$
=\sum_{\substack{l_{p} \geq a_{p}^{*}, a \geq \max \left\{a_{p}, a_{p}^{*}\right\} \\=: \Sigma_{1}+\Sigma_{2}+\Sigma_{3},}}^{f_{p}(s, w ; \psi)} \sum_{0 \leq l_{p}<a_{p}^{*}, a \geq \max \left\{a_{p}, a_{p}^{*}\right\}}+\sum_{l_{p} \geq 0, a_{p} \leq a<\max \left\{a_{p}, a_{p}^{*}\right\}}
$$

where the summands are always $p^{-a s-l_{p} w} \psi(p)^{l_{p}} A\left((-1)^{\alpha-1}\left|T^{*}\right| p^{2 l_{p}}, p^{a} ; \mathfrak{T}\right)$. Equation (6.4) now shows that

$$
\begin{aligned}
\Sigma_{1}= & \kappa \psi(p)^{a_{p}^{*}} p^{-a_{p}^{*} w-(s-m+1) \max \left\{a_{p}, a_{p}^{*}\right\}}\left(1-\frac{\psi(p)}{p^{w}}\right)^{-1}\left(1-p^{m-1-s}\right)^{-1} \\
& +\psi(p) p^{m-2 s-w} \\
& \times\left(\Sigma_{1}+\sum_{l_{p}=a_{p}^{*}-1, a \geq \max \left\{a_{p}, a_{p}^{*}\right\}}+\sum_{l_{p} \geq a_{p}^{*}-1, \max \left\{a_{p}, a_{p}^{*}\right\}-2 \leq a<\max \left\{a_{p}, a_{p}^{*}\right\}}\right) .
\end{aligned}
$$


The second and third sum are of the same type as $\Sigma_{2}$ and $\Sigma_{3}$, i.e. the $l_{p^{-}}$ resp. $a$-summation is finite. With the same reasoning as in [5], bottom of p. 39, it follows that sums of type $\Sigma_{2}$ become polynomials in $p^{-s}$ and $\psi(p) p^{-w}$ after multiplication with $\left(1-p^{m-1-s}\right)$. With the exception of finitely many terms sums of type $\Sigma_{3}$ are geometric sums. Thus they become polynomials in $p^{-s}$ and $\psi(p) p^{-w}$ after multiplication with $\left(1-\psi(p) p^{-w}\right)$. Thus (6.6) and (6.5) show that

$$
\begin{array}{r}
f_{p}(s, w ; \psi)\left(1-p^{m-1-s}\right)\left(1-\psi(p) p^{-w}\right)\left(1-\psi(p) p^{m-2 s-w}\right) \\
=R_{p}\left(p^{-s}, \psi(p) p^{-w}\right)
\end{array}
$$

with $R_{p}(x, y) \in \mathbb{Q}[x, y]$ depending only on $p, b$ and $\mathfrak{T}$. Obviously for $p \nmid 2 T b$, we have $R_{p}(x, y)=\left(1+p^{(m-1) / 2} x\right)\left(1-p^{(m-1) / 2} x y\right)$.

a) For $q \nmid 2 T b$ a prime, $\chi \neq \chi_{0}$ a Dirichlet character modulo $q$ and $\Re s$, $\Re w$ sufficiently large,

$$
\tau_{\alpha}^{*}(s+1, w ; b, \mathfrak{T}, \chi)=\overline{\tau(\chi)}\left|T^{*}\right|^{-w} \chi\left(\left|T^{*}\right|\right) f\left(s, 2 w ; 1, \chi^{2}\right) .
$$

Now (6.1), (6.3) and (6.7) prove the statement of the theorem in this case. If $\chi=\chi_{0} \bmod q$,

$$
\begin{aligned}
& \tau_{\alpha}^{*}\left(s+1, w ; b, \mathfrak{T}, \chi_{0} \bmod q\right) \\
& \quad=\left|T^{*}\right|^{-w} q^{1-2 w} f\left(s, 2 w ; q, \chi_{0} \bmod 1\right)-\left|T^{*}\right|^{-w} f\left(s, 2 w ; 1, \chi_{0} \bmod 1\right) .
\end{aligned}
$$

It follows from (6.3) and (6.2) that

$$
\begin{aligned}
& \sum_{l_{q} \geq 0} q^{-l_{q} w} L_{q}\left(s, q^{l_{q}+1}\right) \\
& \quad=q^{w}\left(f_{q}\left(s, w ; \chi_{0} \bmod 1\right)-L_{q}(s, 1)\right) \\
& \quad=\frac{\left(1+q^{(m-1) / 2-s}\right)\left(1+q^{m-2 s}-q^{(m-1) / 2-s}-q^{m-2 s-w}\right)}{\left(1-q^{m-1-s}\right)\left(1-q^{m-2 s-w}\right)\left(1-q^{-w}\right)} .
\end{aligned}
$$

Again (6.1), (6.3) and (6.7) give the statement of the theorem.

b) For $\chi=\chi_{0} \bmod 1$, we have

$$
\tau_{\alpha}^{*}(s+1, w ; b, \mathfrak{T})=\left|T^{*}\right|^{-w} f\left(s, 2 w ; 1, \chi_{0} \bmod 1\right),
$$

and the statement follows as in a).

Lemma 6.2. Let $m \in \mathbb{N}$ be odd, $\mathfrak{T} \in \mathbb{Z}_{*}^{m \times m}, T:=\operatorname{det} \mathfrak{T}$ and $\alpha \in\{1,2\}$ such that $\alpha \equiv(m+1) / 2+(1-\operatorname{sign}(T)) / 2 \bmod 2$. Set $b:=1, \mathfrak{S}:=\mathfrak{T}$ or $b:=4|T|, \mathfrak{S}:=|T| \mathfrak{T}^{-1}$. Set $S:=\operatorname{det} \mathfrak{S}$. There are polynomials $T_{p}(\cdot ; b, \mathfrak{S}) \in$ $\mathbb{Q}[x]$ for $p \mid 2 T$ with the following properties: 
a) If $q \backslash 2 T$ is a prime and $\chi$ a Dirichlet character modulo $q$, then

$$
\begin{aligned}
h(s, w ; b, \mathfrak{S}, \chi):= & \tau_{\alpha}(s, w ; b, \mathfrak{S}, \chi) P^{*}(s-1 ; 1, \mathfrak{T})(w-1)^{a(\chi(\dot{\bar{q}}))} \\
& \times\left(s+w-2-\frac{m}{2}\right)^{a(\chi(\dot{\bar{q}}))}\left(s-\frac{m}{2}-1\right)^{a(\chi(\dot{\bar{q}}))}
\end{aligned}
$$

is entire with respect to $s$ and $w$, and

$$
\begin{aligned}
& h\left(\frac{m+3}{2}, w ; b, \mathfrak{S}, \chi\right) \\
& =2^{1-a(\chi(\dot{\bar{q}}))} \overline{\tau(\chi)} b^{(m+1) / 2} \chi\left(\left|S^{*}\right|\right)\left|S^{*}\right|^{-w} L\left(2 w, \chi^{2}\right) \\
& \quad \times \prod_{p \mid 2 T} T_{p}\left(\chi^{2}(p) p^{-2 w} ; b, \mathfrak{S}\right)(w-1)^{a(\chi(\dot{\bar{q}}))}\left(w-\frac{1}{2}\right)^{a(\chi(\dot{\bar{q}}))}
\end{aligned}
$$

for $\chi \neq \chi_{0}$, and

$$
\begin{aligned}
& h\left(\frac{m+3}{2}, w ; b, \mathfrak{S}, \chi_{0}\right)= \\
& \quad 2 b^{(m+1) / 2}\left|S^{*}\right|^{-w} \zeta(2 w)\left(q^{1-2 w}-1\right) \prod_{p \mid 2 T} T_{p}\left(p^{-2 w} ; b, \mathfrak{S}\right)
\end{aligned}
$$

for $\chi=\chi_{0}$.

b) The function

$$
\begin{aligned}
& h(s, w ; b, \mathfrak{S}):= \\
& \quad \tau_{\alpha}(s, w ; b, \mathfrak{S}) P^{*}(s-1 ; 1, \mathfrak{T})(w-1)\left(s+w-2-\frac{m}{2}\right)\left(s-\frac{m}{2}-1\right)
\end{aligned}
$$

is entire, and

$$
\begin{aligned}
& h\left(\frac{m+3}{2}, w ; b, \mathfrak{S}\right)= \\
& \quad b^{(m+1) / 2}\left|S^{*}\right|^{-w} \zeta(2 w) \prod_{p \mid 2 T} T_{p}\left(p^{-2 w} ; b, \mathfrak{S}\right)(w-1)\left(w-\frac{1}{2}\right) .
\end{aligned}
$$

Proof. It follows immediately from Theorems 4.4 and 4.5 that $h(s, w ; b, \mathfrak{S}, \chi)$ and $h(s, w ; b, \mathfrak{S})$ are entire. From the definition of $\tau_{\alpha}^{*}$ and Lemma 3.2a) it follows that there is some $c>0$ such that

$$
\left(\tau_{\alpha}(s, w ; b, \mathfrak{S}, \chi)-\tau_{\alpha}^{*}(s, w ; b, \mathfrak{S}, \chi)\right) P^{*}(s-1 ; 1, \mathfrak{T})(2 s-m-3)^{-1}
$$




$$
=\sum_{h \geq 1: h^{*} \neq\left|S^{*}\right|} \frac{\lambda(h, \chi)}{h^{w}} L\left(s-1,(-1)^{\alpha-1} h ; b, \mathfrak{S}\right) P^{*}(s-1 ; 1, \mathfrak{T})(2 s-m-3)^{-1}
$$

converges uniformly on every compact subset of $\{\Re s>m / 2+1, \Re w>c\}$. Therefore it defines a holomorphic function $f(s, w)$ on this set, and

$$
\begin{aligned}
& h(s, w ; b, \mathfrak{S}, \chi) \\
&=\tau_{\alpha}^{*}(s, w ; b,\mathfrak{S}, \chi) P^{*}(s-1 ; 1, \mathfrak{T})(w-1)^{a(\chi(\dot{\bar{q}}))} \\
& \times\left(s+w-2-\frac{m}{2}\right)^{a(\chi(\dot{\bar{q}}))}\left(s-\frac{m}{2}-1\right)^{a(\chi(\dot{\bar{q}}))} \\
&+f(s, w)(2 s-m-3)(w-1)^{a(\chi(\dot{\bar{q}}))} \\
& \times\left(s+w-2-\frac{m}{2}\right)^{a(\chi(\dot{\bar{q}}))}\left(s-\frac{m}{2}-1\right)^{a(\chi(\dot{\bar{q}}))} .
\end{aligned}
$$

Thus for $\Re w>c$,

$$
\begin{aligned}
& h\left(\frac{m+3}{2}, w ; b, \mathfrak{S}, \chi\right) \\
& =\left.\tau_{\alpha}^{*}(s, w ; b, \mathfrak{S}, \chi) P^{*}(s-1 ; 1, \mathfrak{T})\right|_{s=(m+3) / 2}(w-1)^{a(\chi(\dot{\bar{q}}))} \\
& \quad \times\left(w-\frac{1}{2}\right)^{a(\chi(\dot{\bar{q}}))} 2^{-a(\chi(\dot{\bar{q}}))}
\end{aligned}
$$

Analogously, for $\Re s>c$,

$$
\begin{aligned}
& h\left(\frac{m+3}{2}, w ; b, \mathfrak{S}\right)= \\
& \left.\quad \tau_{\alpha}^{*}(s, w ; b, \mathfrak{S}, \chi) P^{*}(s-1 ; 1, \mathfrak{T})\right|_{s=(m+3) / 2}(w-1)\left(w-\frac{1}{2}\right) 2^{-1} .
\end{aligned}
$$

Now the formulae in the lemma follow from Lemma 6.1 if we define

$$
T_{p}(x ; b, \mathfrak{S}):=\left(1-p^{-1}\right)\left(1-p^{-1} x\right)^{-1} R_{p}\left(p^{-(m+1) / 2}, x\right) \in \mathbb{Q}(x) .
$$

The formulae hold for all $w$ since the left hand sides are entire. It remains to prove that the $T_{p}$ are polynomials. Assume $T_{p} \not \equiv 0$ for all $p \mid 2 T$. Otherwise we could set $T_{p} \equiv 0$ for all $p \mid 2 T$. The formula in b) gives

$$
\begin{aligned}
h\left(\frac{m+3}{2}, w ; b, \mathfrak{S}\right) b^{-(m+1) / 2}\left|S^{*}\right|^{w} \zeta(2 w)^{-1}(w-1)^{-1}\left(w-\frac{1}{2}\right)^{-1} & =\prod_{p \mid 2 T} T_{p}\left(p^{-2 w} ; b, \mathfrak{S}\right) .
\end{aligned}
$$


The right hand side has poles at most at the points

$$
-\frac{1}{2}+i \frac{\pi n}{\log p}, \quad n \in \mathbb{Z}, p \mid 2 T \text { prime, }
$$

where the left hand side has no poles. Therefore both sides are entire. Let $p_{0} \mid 2 T$ be a prime. Since $\left\{1, \log p / \log p_{0}|p| 2 T, p \neq p_{0}\right\}$ is linearly independent over $\mathbb{Q}$ the sequence

$$
n \mapsto\left(n \log p / \log p_{0}\right)_{p \mid 2 T, p \neq p_{0}}
$$

is uniformly distributed modulo 1 . Thus there is some $n_{0} \in \mathbb{Z}$ such that, for $p \mid 2 T, p \neq p_{0}$, the function $T_{p}\left(p^{-2 w} ; b, \mathfrak{S}\right)$ is holomorphic and has no zero at $-1 / 2+i \pi n_{0} / \log p_{0}$. Thus $T_{p_{0}}\left(p_{0}^{-2 w} ; b, \mathfrak{S}\right)$ is holomorphic at this point and therefore all $T_{p}(x ; b, \mathfrak{S})$ are polynomials.

Now we show that, for the other possible choice of $\alpha$, the function $\tau_{\alpha}$ has no pole at $s=(m+3) / 2$.

Lemma 6.3. Let $m \in \mathbb{N}$ be odd, $\mathfrak{T} \in \mathbb{Z}_{*}^{m \times m}, T:=\operatorname{det} \mathfrak{T}$ and $\alpha \in\{1,2\}$ such that $\alpha \equiv(m-1) / 2+(1-\operatorname{sign}(T)) / 2 \bmod 2$. Set $b:=1, \mathfrak{S}:=\mathfrak{T}$ or $b:=4|T|, \mathfrak{S}:=|T| \mathfrak{T}^{-1}$. Set $S:=\operatorname{det} \mathfrak{S}$.

a) For $q \backslash 2 T$ a prime and $\chi$ a Dirichlet character modulo $q$, the function (4.4) is still entire after division by $(s-(m+3) / 2)$.

b) The function (4.8) is still entire after division by $(s-(m+3) / 2)$.

Proof. From the special choice of $\alpha$ and Lemma 3.2a) it follows that there is some $c>0$ such that

$$
\begin{aligned}
\tau_{\alpha} & (s, w ; b, \mathfrak{S}, \chi) P^{*}(s-1 ; 1, \mathfrak{T})\left(s-\frac{m+3}{2}\right)^{-1} \\
& =\sum_{h \geq 1} \frac{\lambda(h, \chi)}{h^{w}} L\left(s-1,(-1)^{\alpha-1} h ; b, \mathfrak{S}\right) P^{*}(s-1 ; 1, \mathfrak{T})\left(s-\frac{m+3}{2}\right)^{-1}
\end{aligned}
$$

converges uniformly on every compact subset of $\{\Re s>m / 2+1, \Re w>$ $c$. Therefore it defines a holomorphic function on this set. Consequently (4.4), after division by $(s-(m+3) / 2)$, is still holomorphic on this set. An application of Lemma 4.2 finishes the proof of a). The proof of b) is completely analogous. 
The next lemma contains some sort of functional equation which connects the polynomials $T_{p}(x ; 1, \mathfrak{T})$ and $T_{p}\left(x ; 4|T|,|T| \mathfrak{T}^{-1}\right)$.

Lemma 6.4. Let $m \in \mathbb{N}$ be odd, $\mathfrak{T} \in \mathbb{Z}_{*}^{m \times m}$ and $T:=\operatorname{det} \mathfrak{T}$. Set $\lambda:=(1 \pm \operatorname{sign}(T)) / 4$ where the sign is chosen so that $\lambda \in \mathbb{Z}$. Then for $p \mid 2 T$ prime,

$$
T_{p}(x ; 1, \mathfrak{T})=c_{p} x^{d_{p}} T_{p}\left(p^{-1} x^{-1} ; 4|T|,|T| \mathfrak{T}^{-1}\right), \quad d_{p}:=\operatorname{ord}_{p}\left|T / T^{*}\right|^{1 / 2}
$$

where

$$
\prod_{p \mid 2 T} c_{p}=(-1)^{\lambda}(2|T|)^{(3-m) / 2}
$$

Proof. Choose $\alpha \in\{1,2\}$ with $\alpha \equiv(m+1) / 2+(1-\operatorname{sign}(T)) / 2 \bmod 2$. Multiply both sides of the functional equation in Theorem 4.5 with

$$
P^{*}(s-1 ; 1, \mathfrak{T})(w-1)\left(s+w-2-\frac{m}{2}\right)\left(s-\frac{m}{2}-1\right)\left(s+w-\frac{m}{2}-1\right) w
$$

and plug $s=(m+3) / 2$ in. Lemmas $6.2 \mathrm{~b})$ and 6.3 give

$$
\begin{aligned}
& h\left(\frac{m+3}{2}, w ; 1, \mathfrak{T}\right)\left(w+\frac{1}{2}\right) w= \\
& \quad(2 \pi)^{2 w-1 / 2}(4|T|)^{1 / 2-w} \Gamma(1-w) \Gamma\left(\frac{1}{2}-w\right) 2^{-3 m / 2}|T|^{1 / 2-m} \pi^{-1} \\
& \quad \times \sin \pi(w-\lambda) h\left(\frac{m+3}{2}, \frac{1}{2}-w ; 4|T|,|T| \mathfrak{T}^{-1}\right)(w-1)\left(w-\frac{1}{2}\right)
\end{aligned}
$$

and consequently

$$
\prod_{p \mid 2 T} \frac{T_{p}\left(p^{-2 w} ; 1, \mathfrak{T}\right)}{T_{p}\left(p^{2 w-1} ; 4|T|,|T| \mathfrak{T}^{-1}\right)}=(-1)^{\lambda}\left|\frac{T^{*}}{T}\right|^{w}(2|T|)^{(3-m) / 2} .
$$

In the last step the functional equation of the Riemann zeta function was used and it was assumed that none of the $T_{p}$ vanishes identically. Otherwise we could set $T_{p}: \equiv 0$ for all $p \mid 2 T$. Since the right hand side is entire and has no zeros, an argument similar to that in the proof of Lemma 6.2 shows that each single factor on the left hand side is entire without zeros. Therefore

$$
\frac{T_{p}(x ; 1, \mathfrak{T})}{T_{p}\left(p^{-1} x^{-1} ; 4|T|,|T| \mathfrak{T}^{-1}\right)}=c_{p} x^{d_{p}}
$$


with $c_{p} \in \mathbb{C} \backslash\{0\}$ and $d_{p} \in \mathbb{Z}$ for all $p \mid 2 T$. Plugging this representation back into (6.8) gives

$$
\prod_{p \mid 2 T} c_{p}\left(\prod_{p \mid 2 T} p^{-2 d_{p}}\right)^{w}=(-1)^{\lambda}(2|T|)^{(3-m) / 2}\left|\frac{T^{*}}{T}\right|^{w}
$$

from which the lemma follows.

The functional equation of $\tau_{\alpha}$ is now contained in

Proposition 6.5. Let $m \in \mathbb{N}$ be odd, $\mathfrak{T} \in \mathbb{Z}_{*}^{m \times m}, T:=\operatorname{det} \mathfrak{T}$ and $\alpha \in\{1,2\}$ such that $\alpha \equiv(m-1) / 2+(1-\operatorname{sign}(T)) / 2 \bmod 2$. Set $b:=1$, $\mathfrak{S}:=\mathfrak{T}$ or $b:=4|T|, \mathfrak{S}:=|T| \mathfrak{T}^{-1}$. Set $S:=\operatorname{det} \mathfrak{S}$ and $\lambda:=(1 \pm \operatorname{sign}(\mathfrak{T})) / 4$ where the sign is chosen so that $\lambda \in \mathbb{Z}$.

a) Let $q \times 2 T$ be a prime and $\chi$ a Dirichlet character modulo $q$. Then the function

$$
D_{\alpha}\left(\frac{m+3}{2}, w ; b, \mathfrak{S}, \chi\right)
$$

is meromorphic on $\mathbb{C}$. If $\chi \neq\left(\frac{\dot{q}}{q}\right)$ then it is entire. If $\chi=(\dot{q})$ then it has only a simple pole at $w=1$ with residue $b^{m}(q-1) q^{-3 / 2} \epsilon_{q}\left(\frac{|S|}{q}\right)$ and a simple pole at $w=1 / 2$ with residue

$$
-\left(\frac{|S|}{q}\right) \frac{3}{2 \pi}\left|S^{*}\right|^{-1 / 2} b^{(m+3) / 2} \epsilon_{q} q^{-1 / 2}(q-1) \prod_{p \mid 2 T}\left(1-p^{-2}\right)^{-1} T_{p}\left(p^{-1} ; b, \mathfrak{S}\right) .
$$

Furthermore,

$$
\begin{aligned}
& D_{\alpha}\left(\frac{m+3}{2}, w ; 1, \mathfrak{T}, \chi\right)=C(\bar{\chi}, \alpha, \mathfrak{T}) 2^{-3 m / 2-2}|T|^{-1 / 2-m} \pi^{-1} \chi(-1) \\
& \times\left(\frac{2 \pi}{q}\right)^{2 w-1 / 2}(4|T|)^{1 / 2-w} \Gamma(1-w) \Gamma\left(\frac{1}{2}-w\right)(-1)^{\lambda} \\
& \times\left[\sin \pi\left(w-\frac{1}{2}\right) D_{\alpha}\left(\frac{m+3}{2}, \frac{1}{2}-w ; 4|T|,|T| \mathfrak{T}^{-1}, \bar{\chi}\left(\frac{\dot{q}}{q}\right)\right)\right. \\
& \quad-\frac{3}{\pi}(4|T|)^{(m+3) / 2} \prod_{p \mid 2 T}\left(1-p^{-2}\right)^{-1} T_{p}\left(\bar{\chi}^{2}(p) p^{2 w-1} ; 4|T|,|T| \mathfrak{T}^{-1}\right) \\
& \left.\times \mathcal{L}\left(\frac{1}{2}-w, \bar{\chi}\left(\frac{\dot{-}}{q}\right)\right)\right],
\end{aligned}
$$


where

$$
\mathcal{L}(w, \chi):=\sum_{n \geq 1} \frac{\lambda\left(-n^{2}, \chi\right)}{n^{2 w}}=\left\{\begin{array}{ll}
\tau(\bar{\chi}) L\left(2 w, \chi^{2}\right) & , \quad \chi \neq \chi_{0} \\
\left(q^{1-2 w}-1\right) \zeta(2 w) & , \quad \chi=\chi_{0} .
\end{array}\right\} .
$$

b) The function $D_{\alpha}((m+3) / 2, w ; b, \mathfrak{S})$ is meromorphic on $\mathbb{C}$. It has only one simple pole at $w=1$ with residue $b^{m}$ and another simple pole at $w=1 / 2$ with residue

$$
-\frac{3}{2 \pi}\left|S^{*}\right|^{-1 / 2} b^{(m+3) / 2} \prod_{p \mid 2 T}\left(1-p^{-2}\right)^{-1} T_{p}\left(p^{-1} ; b, \mathfrak{S}\right) .
$$

Furthermore,

$$
\begin{aligned}
& D_{\alpha}\left(\frac{m+3}{2}, w ; 1, \mathfrak{T}\right)= \\
& 2^{-3(m+1) / 2}|T|^{-m-w} \pi^{-3 / 2+2 w} \Gamma(1-w) \Gamma\left(\frac{1}{2}-w\right)(-1)^{\lambda} \\
& \quad \times\left[\sin \pi\left(w-\frac{1}{2}\right) D_{\alpha}\left(\frac{m+3}{2}, \frac{1}{2}-w ; 4|T|,|T| \mathfrak{T}^{-1}\right)\right. \\
& \left.\quad-\frac{3}{\pi}(4|T|)^{(m+3) / 2} \prod_{p \mid 2 T}\left(1-p^{-2}\right)^{-1} T_{p}\left(p^{2 w-1} ; 4|T|,|T| \mathfrak{T}^{-1}\right) \zeta(1-2 w)\right] .
\end{aligned}
$$

Finally, both $D_{\alpha}((m+3) / 2, w ; b, \mathfrak{S}, \chi)$ and $D_{\alpha}((m+3) / 2, w ; b, \mathfrak{S})$ are subject to the growth estimates in Proposition 5.1.

Proof. From Lemma 6.3 it follows that

$$
\left(D_{\alpha}\left(\frac{m+3}{2}, w ; b, \mathfrak{S}, \chi\right)-\frac{a(\chi(\dot{\bar{q}}))}{w-1} b^{m}(q-1) q^{-3 / 2} \epsilon_{q}\left(\frac{|S|}{q}\right)\right)\left(w-\frac{1}{2}\right)^{a(\chi(\dot{\bar{q}}))}
$$

and

$$
\left(D_{\alpha}\left(\frac{m+3}{2}, w ; b, \mathfrak{S}\right)-\frac{1}{w-1} b^{m}\right)\left(w-\frac{1}{2}\right)
$$

are entire. We multiply both sides of the functional equation in Theorem 4.4 with

$$
\begin{aligned}
P^{*}(s- & 1 ; 1, \mathfrak{T})(2 s-m-3)^{-1} \\
& \times\left[(w-1)\left(s+w-2-\frac{m}{2}\right)\left(s-\frac{m}{2}-1\right)\left(s+w-\frac{m}{2}-1\right) w\right]^{a(\chi)}
\end{aligned}
$$


and plug $s=(m+3) / 2$ in. After dividing both sides by common factors in $w$ we get

$$
\begin{aligned}
& D_{\alpha}\left(\frac{m+3}{2}, w ; 1, \mathfrak{T}, \chi\right)= \\
& \quad C(\bar{\chi}, \alpha, \mathfrak{T})\left(\frac{2 \pi}{q}\right)^{2 w-1 / 2}(4|T|)^{1 / 2-w} \Gamma(1-w) \Gamma\left(\frac{1}{2}-w\right) \\
& \times \chi(-1) 2^{-3 m / 2}|T|^{1 / 2-m} \pi^{-1}(-1)^{\lambda} \\
& \quad \times\left[\sin \pi\left(w-\frac{1}{2}\right) \frac{1}{4|T|} D_{\alpha}\left(\frac{m+3}{2}, \frac{1}{2}-w ; 4|T|,|T| \mathfrak{T}^{-1}, \bar{\chi}\left(\frac{\cdot}{q}\right)\right)\right. \\
& \quad-\chi(-1)\left(\frac{-1}{q}\right) \frac{3}{2 \pi} h\left(\frac{m+3}{2}, \frac{1}{2}-w ; 4|T|,|T| \mathfrak{T}^{-1}, \bar{\chi}\left(\frac{\cdot}{q}\right)\right) \\
& \left.\quad \times \prod_{p \mid 2 T}\left(1-p^{-2}\right)^{-1}\left(\frac{1}{2}\left(w+\frac{1}{2}\right) w\right)^{-a(\chi)}\right] .
\end{aligned}
$$

From Lemma 6.2a) the first functional equation now follows. For $\chi \neq(\dot{q})$, the function $D_{\alpha}((m+3) / 2, w ; b, \mathfrak{S}, \chi)$ is entire. For $\chi=(\dot{q})$ it has only poles at $w=1$ and $w=1 / 2$ which are simple. Since the function (6.9) is entire the residues can be computed from the functional equation.

The proof of $\mathrm{b}$ ) is similar and uses Theorem 4.5. The growth estimates follow as in Proposition 5.1.

\section{$\S 7$. Construction of an automorphic function}

Now the procedure basically is as in Section 5 with Proposition 5.1 replaced by Proposition 6.5. Since the functional equations now have an additional term Weil's converse theorem cannot be applied directly. The idea is to bring part of the additional term to the left hand side in order to get a more symmetric functional equation. The following lemma is essential in this procedure.

On $\{\Re s>0\}$, the function

$$
F(s):=\int_{1}^{\infty} u^{-3 / 2}(2 u-1)^{1 / 2-s} d u
$$

is holomorphic.

LEMMA 7.1. $F(s)$ can be continued meromorphically to $\mathbb{C}$ and has the functional equation

$$
F(s)+F\left(\frac{3}{2}-s\right)=\sqrt{\frac{8}{\pi}} \Gamma(s) \Gamma\left(\frac{3}{2}-s\right) .
$$


In particular, $F(1 / 2)=2$ and $F(1)=\sqrt{8}-2$.

Proof. Let $0<\Re s<3 / 2$. Substituting $u^{-1}$ for $u$ in (7.1) gives

$$
F(s)=\int_{0}^{1} u^{s-1}(2-u)^{1 / 2-s} d u .
$$

Substituting $2-u$ for $u$ and $3 / 2-s$ for $s$ gives

$$
F\left(\frac{3}{2}-s\right)=\int_{1}^{2} u^{s-1}(2-u)^{1 / 2-s} d u .
$$

Adding (7.2) and (7.3) and using the relation between the Beta and the Gamma function gives the functional equation for $0<\Re s<3 / 2$. This in turn proves that $F$ can be continued meromorphically to $\mathbb{C}$.

For $t \geq 0$, define

$$
\gamma(t):=\int_{1}^{\infty} u^{-3 / 2} e^{-u t} d u .
$$

Lemma 7.2. The function $\gamma$ is $C^{\infty}$ on $\mathbb{R}^{+}$, continuous on $\mathbb{R}_{0}^{+}$and it solves the initial value problem

$$
\gamma^{\prime}(t)-\frac{1}{2 t} \gamma(t)+\frac{1}{t} e^{-t}=0, \quad \gamma(0)=2
$$

For $\delta, y>0$, we have

$$
\frac{1}{2 \pi i} \int_{\delta-i \infty}^{\delta+i \infty} y^{-w} F\left(w+\frac{1}{2}\right) \Gamma(w) d w=e^{y} \gamma(2 y)
$$

Proof. The differential equation follows by integration by parts. The second formula follows from Fubini's theorem and Mellin's inversion formula for the Gamma function.

Now we can prove Theorem 1.2. Under the assumptions of this theorem, choose $\alpha \in\{1,2\}$ with $\alpha \equiv(m-1) / 2+(1-\operatorname{sign}(T)) / 2 \bmod 2$. The functional equation of the zeta- and L-functions and Proposition 5.2 in [6] show that for all primes $q \backslash 2 T$ and Dirichlet characters $\chi$ modulo $q$, we have the functional equation

$$
\left(\frac{\pi}{q}\right)^{-(1-w)} \Gamma(1-w) \mathcal{L}\left(1-w, \bar{\chi}\left(\frac{\dot{q}}{q}\right)\right)=
$$




$$
\bar{\chi}(-4) \epsilon_{q}\left(\frac{\pi}{q}\right)^{-(w-1 / 2)} \Gamma\left(w-\frac{1}{2}\right) \mathcal{L}\left(w-\frac{1}{2}, \chi\right)
$$

Define

$$
\begin{aligned}
\Lambda(w ; b, \mathfrak{S}, \chi):= & \\
\left(\frac{\pi}{q|T|^{1 / 2}}\right)^{-w} & \left(\Gamma(w) D_{\alpha}\left(\frac{m+3}{2}, w-\frac{1}{2} ; b, \mathfrak{S}, \chi\right)\right. \\
+ & \frac{3 b^{(m+3) / 2}}{(2 \pi)^{3 / 2}} \chi\left(\left|S^{*}\right|\right)\left|S^{*}\right|^{1 / 2-w} F(w) \Gamma\left(w-\frac{1}{2}\right) \mathcal{L}\left(w-\frac{1}{2}, \chi\right) \\
& \left.\times \prod_{p \mid 2 T}\left(1-p^{-2}\right)^{-1} T_{p}\left(\chi^{2}(p) p^{1-2 w} ; b, \mathfrak{S}\right)\right) .
\end{aligned}
$$

Then Proposition 6.5a), Lemmas 6.4 and 7.1 and (7.5) give the functional equation

$$
\begin{aligned}
\Lambda(w ; 1, \mathfrak{T}, \chi)=|T|^{-1 / 4-m} & 2^{-3(m+1) / 2}(-1)^{\lambda+1} \chi(-1) C(\bar{\chi}, \alpha, \mathfrak{T}) \\
& \times \Lambda\left(\frac{3}{2}-w ; 4|T|,|T| \mathfrak{T}^{-1}, \bar{\chi}\left(\frac{\dot{-}}{q}\right)\right) .
\end{aligned}
$$

If $\chi \neq \chi_{0},(\dot{\bar{q}})$, both sides of the equation are entire. If $\chi=(\dot{\dot{q}})$, the left hand side has a simple pole at $w=3 / 2$ with residue

$$
\frac{1}{2 \pi}|T|^{3 / 4}(q-1) \epsilon_{q}\left(\frac{|T|}{q}\right)
$$

and a simple pole at $w=1$ with residue

$$
-\frac{3}{\sqrt{8} \pi^{2}} q^{1 / 2} \epsilon_{q}(q-1)\left|\frac{T}{T^{*}}\right|^{1 / 2}\left(\frac{|T|}{q}\right) \prod_{p \mid 2 T}\left(1-p^{-2}\right)^{-1} T_{p}\left(p^{-1} ; 1, \mathfrak{T}\right) .
$$

If $\chi=\chi_{0}$, the left hand side has a simple pole at $w=1 / 2$ with residue

$$
\frac{3}{\pi^{2}} 2^{-m / 2}|T|^{-m / 2+7 / 4}(-1)^{\lambda+1} q^{1 / 2}(q-1) \prod_{p \mid 2 T}\left(1-p^{-2}\right)^{-1} T_{p}\left(p^{-1} ; 4|T|,|T| \mathfrak{T}^{-1}\right)
$$

and a simple pole at $w=0$ with residue

$$
\frac{1}{\pi} 2^{(m-5) / 2}|T|^{1 / 2}(-1)^{\lambda}(q-1) .
$$


Furthermore, the function $\Lambda(w ; 1, \mathfrak{T}, \chi)$ grows at most polynomially in vertical strips of finite width.

Similarly, define

$$
\begin{aligned}
\Lambda(w ; b, \mathfrak{S}):=\left(\frac{\pi}{|T|^{1 / 2}}\right)^{-w}\left(\Gamma(w) D_{\alpha}\left(\frac{m+3}{2}, w-\frac{1}{2} ; b, \mathfrak{S}\right)\right. \\
+\frac{3 b^{(m+3) / 2}}{(2 \pi)^{3 / 2}}\left|S^{*}\right|^{1 / 2-w} F(w) \Gamma\left(w-\frac{1}{2}\right) \zeta(2 w-1) \\
\left.\quad \times \prod_{p \mid 2 T}\left(1-p^{-2}\right)^{-1} T_{p}\left(p^{1-2 w} ; b, \mathfrak{S}\right)\right) .
\end{aligned}
$$

Then

$$
\Lambda(w ; 1, \mathfrak{T})=|T|^{-1 / 4-m} 2^{-3(m+1) / 2}(-1)^{\lambda+1} \Lambda\left(\frac{3}{2}-w ; 4|T|,|T| \mathfrak{T}^{-1}\right) .
$$

The left hand side is meromorphic on $\mathbb{C}$ and has only simple poles at $w=$ $3 / 2,1,1 / 2,0$ with the respective residues

$$
\begin{aligned}
& \frac{1}{2 \pi}|T|^{3 / 4}, \quad-\frac{3}{\sqrt{8} \pi^{2}}\left|\frac{T}{T^{*}}\right|^{1 / 2} \prod_{p \mid 2 T}\left(1-p^{-2}\right)^{-1} T_{p}\left(p^{-1} ; 1, \mathfrak{T}\right), \\
& \frac{3}{\pi^{2}} 2^{-m / 2}|T|^{-m / 2+7 / 4}(-1)^{\lambda+1} \prod_{p \mid 2 T}\left(1-p^{-2}\right)^{-1} T_{p}\left(p^{-1} ; 4|T|,|T| \mathfrak{T}^{-1}\right), \\
& \frac{1}{\pi} 2^{(m-5) / 2}|T|^{1 / 2}(-1)^{\lambda} .
\end{aligned}
$$

It grows at most polynomially in vertical strips of finite width. Define the numbers $a_{n}(b, \mathfrak{S}), n \geq 1$, implicitly by

$$
\sum_{n \geq 1} \frac{a_{n}(b, \mathfrak{S})}{n^{s}}=\zeta(s) \prod_{p \mid 2 T}\left(1-p^{-2}\right)^{-1} T_{p}\left(p^{-s} ; b, \mathfrak{S}\right) .
$$

For $z=x+i y \in \mathbb{H}$, define

$$
\begin{aligned}
\mathcal{H}(z ; b, \mathfrak{S}):= & \pi^{-1} 2^{(m-5) / 2} b^{(m+3) / 2}|S|^{1 / 2}(-1)^{\lambda+1} \\
& +\sum_{n \geq 1} \mathcal{S}\left(\frac{m+3}{2},(-1)^{(m+1) / 2} \operatorname{sign}(T) n ; b, \mathfrak{S}\right) n^{1 / 2} e^{2 \pi i n z} \\
\mathcal{K}(z ; b, \mathfrak{S}):= & \frac{3}{(2 \pi)^{2}} b^{(m+3) / 2} y^{-1 / 2} \prod_{p \mid 2 T}\left(1-p^{-2}\right)^{-1} T_{p}(1 ; b, \mathfrak{S})
\end{aligned}
$$




$$
+\frac{3 b^{(m+3) / 2}}{(2 \pi)^{2}} y^{-1 / 2} \sum_{n \geq 1} a_{n}(b, \mathfrak{S}) \gamma\left(4 \pi\left|S^{*}\right| n^{2} y\right) e^{-2 \pi i\left|S^{*}\right| n^{2} z}
$$

Since the functions $\Lambda(w ; b, \mathfrak{S})$ and $\Lambda(w ; b, \mathfrak{S}, \chi)$ contain the additional function $F(w)$ we cannot apply Weil's converse theorem directly. But its proof can be imitated. The first step is to compute

$$
\frac{1}{2 \pi i} \int_{\delta-i \infty}^{\delta+i \infty}\left(2|T|^{1 / 2} y\right)^{-w} \Lambda(w ; 1, \mathfrak{T}) d w
$$

for $\delta>3 / 2, y>0$, in two ways. Firstly the defining series for $\Lambda(w ; 1, \mathfrak{T})$ is plugged in and Mellin's inversion formula and Lemma 7.2 are used. Secondly the line of integration is moved to $\Re w=\kappa<0$ with the help of the Phragmen-Lindelöf principle. Here the residues are taken into account and the functional equation (7.7) is used. Thus the defining series can be used again. Equating both expressions one gets

$$
\begin{aligned}
\mathcal{H}(i y & ; 1, \mathfrak{T})+\mathcal{K}(i y ; 1, \mathfrak{T})=|T|^{-m-1} 2^{-3 m / 2-3}(-1)^{\lambda+1} y^{-3 / 2} \\
& \times\left[\mathcal{H}\left(-\frac{1}{4|T| i y} ; 4|T|,|T| \mathfrak{T}^{-1}\right)+\mathcal{K}\left(-\frac{1}{4|T| i y} ; 4|T|,|T| \mathfrak{T}^{-1}\right)\right]
\end{aligned}
$$

for $y>0$. From (7.8) it follows that for $q \backslash 2 T$ a prime and $\chi$ a Dirichlet character modulo $q$,

$$
\begin{aligned}
& \sum_{n \geq 1} \frac{a_{n}(b, \mathfrak{S}) \lambda\left(-n^{2}, \chi\right)}{n^{2 w-1}}= \\
& \quad \mathcal{L}\left(w-\frac{1}{2}, \chi\right) \prod_{p \mid 2 T}\left(1-p^{-2}\right)^{-1} T_{p}\left(\chi^{2}(p) p^{1-2 w} ; b, \mathfrak{S}\right) .
\end{aligned}
$$

For $z \in \mathbb{H}$, define

$$
\begin{aligned}
& \mathcal{H}(z ; b, \mathfrak{S}, \chi):= \\
& \pi^{-1} 2^{(m-5) / 2} b^{(m+3) / 2}|S|^{1 / 2}(-1)^{\lambda+1} \lambda(0, \chi) \\
& \quad+\sum_{n \geq 1} \mathcal{S}\left(\frac{m+3}{2},(-1)^{(m+1) / 2} \operatorname{sign}(T) n ; b, \mathfrak{S}\right) n^{1 / 2} \lambda(n, \chi) e^{2 \pi i n z} \\
& \mathcal{K}(z ; b, \mathfrak{S}, \chi):= \\
& \frac{3}{(2 \pi)^{2}} b^{(m+3) / 2} y^{-1 / 2} \prod_{p \mid 2 T}\left(1-p^{-2}\right)^{-1} T_{p}(1 ; b, \mathfrak{S}) \lambda(0, \chi)
\end{aligned}
$$




$$
+\frac{3 b^{(m+3) / 2}}{(2 \pi)^{2}} y^{-1 / 2} \sum_{n \geq 1} a_{n}(b, \mathfrak{S}) \gamma\left(4 \pi\left|S^{*}\right| n^{2} y\right) \lambda\left(-\left|S^{*}\right| n^{2}, \chi\right) e^{-2 \pi i\left|S^{*}\right| n^{2} z}
$$

The same procedure as above gives

$$
\begin{aligned}
& \mathcal{H}\left(i q^{-1} y ; 1, \mathfrak{T}, \chi\right)+\mathcal{K}\left(i q^{-1} y ; 1, \mathfrak{T}, \chi\right) \\
&=|T|^{-m-1} 2^{-3 m / 2-3}(-1)^{\lambda+1} \chi(-1) C(\bar{\chi}, \alpha, \mathfrak{T}) y^{-3 / 2} \\
& \times\left[\mathcal{H}\left(-\frac{1}{4|T| q i y} ; 4|T|,|T| \mathfrak{T}^{-1}, \bar{\chi}\left(\frac{\dot{q}}{q}\right)\right)\right. \\
&\left.\quad+\mathcal{K}\left(-\frac{1}{4|T| q i y} ; 4|T|,|T| \mathfrak{T}^{-1}, \bar{\chi}\left(\frac{\cdot}{q}\right)\right)\right] .
\end{aligned}
$$

We would like to conclude now that similar relations hold for arbitrary $z \in \mathbb{H}$ instead of $i y \in i \mathbb{R}^{+}$. In the case of holomorphic modular forms this is done with the identity principle. In the case of Maaß wave forms the hyperbolic Laplace equation is used. Here we need the lemma below. For $z \in \mathbb{H}$, define

$$
\begin{gathered}
\Theta(z ; b, \mathfrak{S}):=\frac{3 b^{(m+3) / 2}}{(2 \pi)^{2}}\left(\sum_{n \geq 1} a_{n}(b, \mathfrak{S}) e^{2 \pi i\left|S^{*}\right| n^{2} z}\right. \\
\left.\quad+\frac{1}{2} \prod_{p \mid 2 T}\left(1-p^{-2}\right)^{-1} T_{p}(1 ; b, \mathfrak{S})\right), \\
\Theta(z ; b, \mathfrak{S}, \chi):=\frac{3 b^{(m+3) / 2}}{(2 \pi)^{2}}\left(\sum_{n \geq 1} a_{n}(b, \mathfrak{S}) \lambda\left(-\left|S^{*}\right| n^{2}, \chi\right) e^{2 \pi i\left|S^{*}\right| n^{2} z}\right. \\
\left.\quad+\frac{1}{2} \prod_{p \mid 2 T}\left(1-p^{-2}\right)^{-1} T_{p}(1 ; b, \mathfrak{S}) \lambda(0, \chi)\right) .
\end{gathered}
$$

LEMMA 7.3. $\Theta(z ; b, \mathfrak{S})$ is a modular form of weight $1 / 2$ on $\Gamma_{0}(4|T|)$ with respect to the theta multiplier system and character $\chi_{4\left|S^{*}\right|}$. Furthermore,

$$
\begin{aligned}
& |T|^{-m}(-1)^{\lambda} 2^{-3 m / 2-1} \Theta\left(z ; 4|T|,|T| \mathfrak{T}^{-1}\right)=(-i z)^{-1 / 2} \Theta\left(-\frac{1}{4|T| z} ; 1, \mathfrak{T}\right) \\
& |T|^{-m}(-1)^{\lambda} 2^{-3 m / 2-1} \chi(-1) C(\bar{\chi}, \alpha, \mathfrak{T}) \Theta\left(z ; 4|T|,|T| \mathfrak{T}^{-1}, \bar{\chi}\left(\frac{\cdot}{q}\right)\right) \\
& =(-i q z)^{-1 / 2} \Theta\left(-\frac{1}{4|T| q^{2} z} ; 1, \mathfrak{T}, \chi\right)
\end{aligned}
$$




$$
\begin{aligned}
& \frac{\partial}{\partial \bar{z}} \mathcal{K}(z ; b, \mathfrak{S})=\frac{1}{2 i} y^{-3 / 2} \Theta(-\bar{z} ; b, \mathfrak{S}), \\
& \frac{\partial}{\partial \bar{z}} \mathcal{K}(z ; b, \mathfrak{S}, \chi)=\frac{1}{2 i} y^{-3 / 2} \Theta(-\bar{z} ; b, \mathfrak{S}, \chi) .
\end{aligned}
$$

Proof. With the notations from Section 5 (where $\kappa_{\alpha l}$ and $\lambda_{\alpha l}$ are replaced by 1 and 0$)$, Lemma 6.4 and the equations (7.5) and (7.10) give

$$
\begin{aligned}
& \Lambda_{4|T|}(w ; \Theta(\cdot ; 1, \mathfrak{T}), \chi) \\
& \quad=|T|^{-m-1 / 4}(-1)^{\lambda} 2^{-3(m+1) / 2} C_{\chi}^{(1)} \Lambda_{4|T|}\left(\frac{1}{2}-w ; \Theta\left(\cdot ; 4|T|,|T| \mathfrak{T}^{-1}\right), \bar{\chi}\left(\frac{\cdot}{q}\right)\right)
\end{aligned}
$$

for $q \backslash 2 T$ a prime and $\chi \neq \chi_{0},(\dot{q})$ a Dirichlet character modulo $q$; both sides are entire. For $\chi=(\dot{\bar{q}})$, we have

$$
\begin{aligned}
& \Lambda_{4|T| q^{2}}\left(w ; \Theta(\cdot ; 1, \mathfrak{T})_{(\dot{q})}\right) \\
& \quad=|T|^{-m-1 / 4}(-1)^{\lambda} 2^{-3(m+1) / 2} C_{\chi}^{(2)} \Lambda_{4|T| q^{2}}\left(\frac{1}{2}-w ; \Theta\left(\cdot ; 4|T|,|T| \mathfrak{T}^{-1}\right)_{q}\right),
\end{aligned}
$$

where the left hand side has only a simple pole at $w=1 / 2$ with the residue

$$
\begin{aligned}
& |T|^{-m-1 / 4} 2^{-3(m+1) / 2}(-1)^{\lambda} \frac{3}{(2 \pi)^{2}}(4|T|)^{(m+3) / 2} C_{\chi}^{(2)}\left(q^{1 / 2}-q^{-1 / 2}\right) \\
& \times \frac{1}{2} \prod_{p \mid 2 T}\left(1-p^{-2}\right)^{-1} T_{p}\left(1 ; 4|T|,|T| \mathfrak{T}^{-1}\right) .
\end{aligned}
$$

Furthermore,

$$
\begin{aligned}
& \Lambda_{4|T|}(w ; \Theta(\cdot ; 1, \mathfrak{T}))= \\
& \quad|T|^{-m-1 / 4}(-1)^{\lambda} 2^{-3(m+1) / 2} \Lambda_{4|T|}\left(\frac{1}{2}-w ; \Theta\left(\cdot ; 4|T|,|T| \mathfrak{T}^{-1}\right)\right),
\end{aligned}
$$

where the left hand side has only simple poles at $w=1 / 2$ and $w=0$ with residues

$$
\begin{aligned}
|T|^{-m-1 / 4} 2^{-3(m+1) / 2} & (-1)^{\lambda} \frac{3}{(2 \pi)^{2}}(4|T|)^{(m+3) / 2} \\
& \times \frac{1}{2} \prod_{p \mid 2 T}\left(1-p^{-2}\right)^{-1} T_{p}\left(1 ; 4|T|,|T| \mathfrak{T}^{-1}\right)
\end{aligned}
$$

and

$$
-\frac{3}{(2 \pi)^{2}} \frac{1}{2} \prod_{p \mid 2 T}\left(1-p^{-2}\right)^{-1} T_{p}(1 ; 1, \mathfrak{T}) .
$$


Now the first statement follows from Weil's converse theorem for half integral weights. The transformation formulae under inversion follow from Hecke's theorem. Since the termwise differentiated series for $\mathcal{K}(z ; b, \mathfrak{S})$ and $\mathcal{K}(z ; b, \mathfrak{S}, \chi)$ converge uniformly on every compact subset of $\mathbb{H}$, these series define $C^{\infty}$ functions. The equations for the partial derivatives now follows from Lemma 7.2.

We also need to know how Möbius transformations and differentiating with respect to $\bar{z}$ interchange. The following lemma follows by a simple computation.

Lemma 7.4. Let $f: \mathbb{H} \rightarrow \mathbb{C}$ be $C^{\infty}$ and $A=\left(\begin{array}{ll}a & b \\ c & d\end{array}\right) \in \mathrm{GL}_{2}^{+}(\mathbb{R})$. Then

$$
\frac{\partial}{\partial \bar{z}}(f(A\langle z\rangle))=\frac{\operatorname{det} A}{(c \bar{z}+d)^{2}} \frac{\partial f}{\partial \bar{z}}(A\langle z\rangle) .
$$

Now set $\mathcal{F}(\cdot ; b, \mathfrak{S}, \chi)=(\mathcal{H}+\mathcal{K})(\cdot ; b, \mathfrak{S}, \chi)$. From $(7.11)$ it follows that

$$
\begin{aligned}
\mathcal{G}(z):=\mathcal{F}(z ; 1, \mathfrak{T}, \chi) & \\
-|T|^{-m-1} 2^{-3 m / 2-3}(-1)^{\lambda+1} \chi(-1) C(\bar{\chi}, \alpha, \mathfrak{T})(-i q z)^{-3 / 2} & \\
& \times \mathcal{F}\left(-\frac{1}{4|T| q^{2} z} ; 4|T|,|T| \mathfrak{T}^{-1}, \bar{\chi}\left(\frac{\cdot}{q}\right)\right)
\end{aligned}
$$

vanishes for $z=i y \in i \mathbb{R}^{+}$. On the other hand, from Lemmas 7.3 and 7.4 it follows that $\partial \mathcal{G} / \partial \bar{z} \equiv 0$ on $\mathbb{H}$. Thus $\mathcal{G}$ is holomorphic on $\mathbb{H}$, and the identity principle gives $\mathcal{G} \equiv 0$ on $\mathbb{H}$. Consequently,

$$
\begin{aligned}
\mathcal{F}(z ; 1, \mathfrak{T}, \chi)= & |T|^{-m-1} 2^{-3 m / 2-3}(-1)^{\lambda+1} \chi(-1) C(\bar{\chi}, \alpha, \mathfrak{T}) \\
& \times(-i q z)^{-3 / 2} \mathcal{F}\left(-\frac{1}{4|T| q^{2} z} ; 4|T|,|T| \mathfrak{T}^{-1}, \bar{\chi}\left(\frac{\dot{z}}{q}\right)\right), \quad z \in \mathbb{H} .
\end{aligned}
$$

Similarly, from $(7.9)$ it follows that for $\mathcal{F}(\cdot ; b, \mathfrak{S})=(\mathcal{H}+\mathcal{K})(\cdot ; b, \mathfrak{S})$, we have

$$
\begin{gathered}
\mathcal{F}(z ; 1, \mathfrak{T})=|T|^{-m-1} 2^{-3 m / 2-3}(-1)^{\lambda+1}(-i z)^{-3 / 2} \mathcal{F}\left(-\frac{1}{4|T| z} ; 4|T|,|T| \mathfrak{T}^{-1}\right) \\
z \in \mathbb{H} .
\end{gathered}
$$

The last two relations are the essential ingredients in the proof of Weil's converse theorem. From this point on everything works as in the case of 
holomorphic Fourier expansions. There is one further situation in which holomorphy is needed. If $A=\left(\begin{array}{ll}a & b \\ c & d\end{array}\right) \in \Gamma_{0}(4|T|)$, then

$$
\left.\mathcal{F}(\cdot ; b, \mathfrak{S})\right|_{3 / 2} A(z)-\chi_{4\left|S^{*}\right|}(d) \mathcal{F}(z ; b, \mathfrak{S})
$$

is holomorphic on $\mathbb{H}$. This can be shown again with Lemmas 7.3 and 7.4. Thus Theorem 1.2 is proved.

\section{REFERENCES}

[1] J.H. Bruinier, Modulformen halbganzen Gewichts und Beziehungen zu Dirichletreihen, Diplomarbeit, Universität Heidelberg, 1997.

[2] H. Cohen, Sums involving the values at negative integers of L-functions of quadratic characters, Math. Ann., 217 (1975), 271-285.

[3] B.A. Datskovsky, On Dirichlet series whose coefficients are class numbers of binary quadratic forms, Nagoya Math. J., 142 (1996), 95-132.

[4] O.T. O'Meara, Introduction to Quadratic Forms, Springer, 1973.

[5] M. Peter, Dirichlet series in two variables, J. Reine Angew. Math., 522 (2000), $27-50$.

[6] G. Shimura, On modular forms of half integral weight, Ann. Math., 97 (1973), 440-481.

[7] T. Shintani, On zeta-functions associated with the vector space of quadratic forms, J. Fac. Sci. Univ. Tokyo Sect. IA Math., 22 (1975), 25-65.

[8] C.L. Siegel, Die Funktionalgleichungen einiger Dirichlet'scher Reihen, Math. Z., 63 (1956), 363-373.

[9] — Über das quadratische Reziprozitätsgesetz in algebraischen Zahlkörpern, Nachr. Akad. Wiss. Göttingen, math.-phys. Kl. 1960, 1-16.

[10] T. Ueno, Elliptic modular forms arising from zeta functions in two variables attached to the space of binary Hermitian forms, J. Number Th., 86 (2001), 302-329.

[11] — Modular forms arising from zeta functions in two variables attached to prehomogeneous vector spaces acted on by maximal parabolic subgroups of orthogonal groups, PhD thesis, Tokyo, 2001.

[12] A. Weil, Über die Bestimmung Dirichlet'scher Reihen durch Funktionalgleichungen, Math. Ann., 168 (1967), 149-156.

[13] D.B. Zagier, Nombres de classes et formes modulaires de poids 3/2, C.R. Acad. Sc. Paris, 281 (1975), 883-886.

Mathematisches Institut

Albert-Ludwigs-Universität

Eckerstr. 1

D-79104 Freiburg

Germany

manfred.peter@math.uni-freiburg.de 